\title{
Qualitative Analyse der fünf NGOs im Dreieck der Handlungsrationalität
}

Die qualitative Analyse gliedert sich entsprechend der kombinierten theorieund materialbasierten Forschungsstrategie (siehe Kapitel 3) in zwei Teilbereiche. Zuerst widmet sich die Untersuchung den theoriegeleiteten Kategorien, die sich aus dem Dreieck der Handlungsrationalität ableiten. Hierunter fallen die die Unterstützungs-, die Einfluss- und die Reputationslogik. Im Anschluss folgen die querliegenden, materialbasierten Kategorien, in denen sich ergänzende Schwerpunkte der Handlungsstrategien finden. Diese umfassen die übergeordnete Strategie, die Themenwahl, die Rolle von Expertise und die Legitimationsargumentation.

Das Interviewmaterial wurde geordnet, zusammengefasst und aggregiert, sodass zu den fünf Fallstudien ein umfängliches Einschätzungsbild präsentiert wird. Dies zeigt sich an den Quellennachweisen der entsprechenden Abschnitte. Bei den Zusammenfassungen für die fünf NGOs wird aufgrund der Anforderungen der Interview-Anonymisierung darauf verzichtet, die Quellen der einzelnen Interviewpartner*innen explizit zu nennen.

\subsection{Auswertung der theoriegeleiteten Kategorie der Unterstützungslogik}

Die Unterstützungslogik unterteilt sich u. a. in die theoriegeleiteten Kategorien Organisationsaufbau, Mitgliederstruktur, Beteiligungsmöglichkeiten, Mitgliederkommunikation und inhaltlicher Basis-Input, welche mit dem Material als Interviews, Dokumenten, Podcasts und weiteren Quellen angefüllt wurden (Abbildung 5.1). 


\section{Unterstützungslogik im Dreieck}

mit Kategorien Organisationsaufbau, Mitgliederstruktur, Beteiligungs-

möglichkeiten, Mitgliederkommunikation und inhaltlicher Basis-Input

(Grafik: eigene Darstellung)

Abbildung 5.1 Hauptkategorie der Unterstützungslogik

Attac ist als eingetragener Verein organisiert (siehe Kapitel 4 für die Debatte um Gemeinnützigkeit). Die Mitgliedschaft als voll stimmberechtigtes Mitglied steht jeder Einzelperson offen. Vergleichbar mit einer Partei oder einem Verband verfügt Attac über dezentrale, föderale Organisationsstrukturen, d. h. es gibt Orts- und Regionalgruppen in ganz Deutschland, bundesweite Arbeitsgruppen und ein zentrales Bundesbüro. Eine Besonderheit für die Organisationsverfassung und das Selbstverständnis von Attac ist, dass sowohl klassische Vollmitglieder als auch lose Unterstützer*innen, die sich punktuell einbringen wollen, gleichbehandelt und als „Attacies“ bezeichnet werden. Trotz der zentralen Rolle, den die Mitgliedsbeiträge bei der Finanzierung der Organisation spielen, tritt dieser Unterschied in seiner Relevanz deutlich hinter die essenzielle Funktion des tatsächlichen ehrenamtlichen Engagements der Aktiven zurück. Die inhaltliche Arbeit von Attac ist laut Intervieweinschätzungen nur möglich, da sich Ehrenamtliche auf verschiedenen Ebenen (Orts- und Regionalgruppen, Bundesebene) einbringen. Wer aktiv werden möchte, kann sich vor Ort einer Attac-Gruppe anschließen oder - unterstützt vom Bundesbüro - selbst eine Gruppe gründen, bzw. sich in den Arbeitszusammenhängen auf der Bundesebene engagieren.

Das Bundesbüro fungiert laut den Aussagen der Interviews nicht als zentralisierte Organisationsspitze, sondern als Dienstleister für Attacies, für Orts- und Regionalgruppen sowie für die bundesweiten Arbeitsgruppen. Trotz des betont basisdemokratischen Aufbaus besteht die Notwendigkeit einer professionellen Infrastruktur für Finanzen, Pressearbeit, Bildungsarbeit und Koordination, die vom Bundesbüro organisiert wird.

Die Beteiligungsmöglichkeiten bei Attac sind divers und essenziell für das Bestehen der Organisation. Durch die Wahrnehmung jedes Engagierten als Attacie ergibt sich ein niedrigschwelliger und flexibler Zugang zur Organisation. Trotz der Betonung der Mitgliederbasis wird auf diese Weise auch ein punktuelles, ungebundenes Engagement ermöglicht. Die Themensetzung und Entscheidung über Schwerpunkte geschehen autonom in den Ortsgruppen. 
Um trotz der gewollt dezentralen Arbeit vor Ort eine Vernetzung sicherzustellen, organisiert das Bundesbüro jährlich Regionalkonferenzen, die einen persönlichen Austausch ermöglichen. Aktive Teilnahme ist darüber hinaus bei Protesten, Demonstrationen oder bei anderen Aktionen möglich. Auch hier richtet sich der Mobilisierungsaufruf explizit an alle Unterstützer*innen und beschränkt sich nicht unbedingt nur auf die Mitglieder.

Die interne Kommunikation der Mitglieder untereinander und innerhalb der Ortsgruppen verläuft unabhängig vom Bundesbüro. Das Bundesbüro informiert alle Interessierten über Rundbriefe und verschickt regelmäßig Mitgliederinformationen. Jede Attac-Gruppe hat eine Gruppenansprechpartner*in, die vom Bundesbüro gesonderte Informationen erhält. Laut Interviewaussagen wurde auch versucht, eine Diskussionsgruppenliste per Mail aufzubauen. Aufgrund der von physischer Präsenz geprägten Organisationskultur setzte sich diese Maßnahme jedoch nicht durch. Attac bespielt darüber hinaus Social Media Kanäle für die Organisationskommunikation.

Die gesamte Organisation ist vollständig bottom-up ausgerichtet, die Aktiven vor Ort bilden das Fundament. Thematische Entscheidungen und Ausrichtungen werden vollständig basisdemokratisch organisiert. Neben der Autonomie der einzelnen Ortsgruppen, wird jährlich zentral über die Organisationsschwerpunktsetzung im Rahmen des Entscheidungsgremiums „Ratschlag“ entschieden (siehe Infobox zu Attac, Abbildung 5.2).

LobbyControl hat die Rechtsform eines eingetragenen, als gemeinnützig anerkannten Vereins und hat sich für den Aufbau einer abgestuften Mitgliedschaft entschieden. So kann jede natürliche Person Fördermitglied werden. Über den Status als Vollmitglied verfügen nur wenige, ausgesuchte Personen (siehe Kapitel 4). Die Fördermitglieder sind laut den Einschätzungen in den Interviews zentral für die finanzielle Unabhängigkeit der Organisation. Die finanzielle Förderung durch juristische Personen, Unternehmen oder Stiftungen wird - ebenfalls aus Gründen der Unabhängigkeit - kritisch gesehen und individuell geprüft. Die abgestuften Beitritts- und damit Partizipationsmöglichkeiten im Verein werden durch die Sorge vor einer möglichen Unterwanderung bzw. einer Lähmung eines kleinen Vereines durch Außenstehende erklärt. Der Organisationsaufbau spiegelt die Entscheidung, sowohl handlungsfähig, schlagfertig als auch finanziell unabhängig zu sein. Die Geschäftsstelle fungiert als Organisationsspitze.

Durch die abgestufte Mitgliedschaft sind die reinen Fördermitglieder in ihrem Engagement jenseits finanzieller Unterstützung eingeschränkt. LobbyControl hat - anders als Attac - keine ehrenamtliche Struktur analog zu föderal aufgebauten, großen Umweltverbänden bzw. Parteien. Angebote an die Fördermitglieder im Besonderen und alle Interessierten im Allgemeinen sind trotzdem vorhanden. 


\section{Von Attacies für Attacies - Richtungsentscheidung in der fluiden Masse}

Attac hat für sich - neben der dezentral basisdemokratischen Ausrichtung - ein inklusives Verständnis von „Mitgliedschaft“ etabliert. „Attacie“ ist, wer sich beteiligt, auch ohne unterschriebenen Mitgliedsantrag. Dies gilt auch bei den zweimal jährlich stattfindenden Versammlungen des „Ratschlags“ - Attacs höchstem Entscheidungsgremium und Mitgliederversammlungsäquivalent. Ein „Ratschlag“ pro Jahr wird explizit als Basistreffen zum Erfahrungsaustausch verstanden. Ein zweiter wird als Wahl- und Entscheidungsgremium durchgeführt (Attac 2019d). Abstimmungen über thematische Schwerpunktsetzungen sind hier für alle anwesenden Attacies möglich. Lediglich die Haushalts- und Finanzfragen werden ausschließlich von gewählten Delegierten der Ortsgruppen entschieden. Alle Entscheidungen sollen im Konsens getroffen werden. Jede Person kann ein Veto einlegen (Attac 2019d).

Der „Ratschlag“ wählt einen sogenannten Rat, der zwischen den „Ratschlägen“ über strategische Fragen, Inhalte und Aktionen entscheiden kann. Daneben wird ein Koordinierungskreis bestimmt, der sich um das politische Alltagsgeschäft kümmert. Die Mitglieder der „AG Büro“ des Koordinierungskreises verstehen sich als Vorgesetzte der Geschäftsstelle. In der Geschäftsstelle sind hauptamtliche Mitarbeiter*innen angestellt, die sich professionell um Fundraising, Öffentlichkeits- und Pressearbeit, aber auch fokussiert um Attacie- und Gruppenunterstützung kümmern. Das Bundesbu ro sieht sich sich als Dienstleister der Basis. Die Basis wiederum begreift die Organisation als partizipatorische Plattform für die inhaltlichen Impulse der Aktiven. Entsprechend hoch gewichtet die Organisationskultur ihre basisdemokratischen Strukturen und Mitbestimmungsmöglichkeiten, die sich explizit gegen eine top-down Funktionsweise stellen.

Abbildung 5.2 Infobox zu Attac

So fungiert die Internetseite von LobbyControl als Informationsplattform. Des Weiteren bietet die Organisation lobbykritische Stadtführungen im Berliner Regierungsviertel an. Auch die Beteiligung an Veranstaltungen und Demonstrationen 
steht allen Interessierten offen. In den vergangenen Jahren gab es die Initiative, einen Stammtisch in Köln und Berlin aufzubauen.

Organisationsintern findet eine kritische Reflexion über die momentane Beteiligungsstruktur und über das Ideal eines basisdemokratischen Organisationsaufbaus statt. Die Struktur der abgestuften Mitgliedschaft wird allerdings in erster Linie als Ressourcenproblem wahrgenommen. Trotz der geringen direkten Beteiligungsmöglichkeiten scheint es eine hohe Identifikation aller Förder- und Vollmitglieder mit der Organisation zu geben, da es laut Interviewaussagen kaum Austritte gibt.

Die Mitglieder- und Interessiertenkommunikation wird über Newsletter, Social Media und die Internetseite gestaltet. Die Social Media Kanäle können als direkte Kommunikation im dialogischen Austausch verstanden werden. Bei Mitgliederversammlungen, Stammtischen oder anderen Arten des Zusammentreffens ist der Austausch mit Unterstützer*innen ebenfalls möglich. Über die digitalen Kanäle werden Vorschläge eingespeist, die den möglichen Themenpool anreichern. Die tatsächliche Themenauswahl findet in der Praxis zentral und top-down entlang der Organisationszielsetzung sowie anhand der entwickelten Expertise statt. Es gibt kein fest institutionalisiertes Feedbackverfahren der Mitglieder und Unterstützer*innen, sondern punktuelle, indirekte Rückkopplung über Nichtaustritte und Eintritte und das thematisch generierte Spendenvolumen.

Campact ist ein eingetragener Verein (siehe Kapitel 4 für Debatte um Gemeinnützigkeit). Satzungsgemäß ist eine abgestufte Mitgliedschaft vorgegeben. Die Zahl der Vollmitglieder ist auf zwölf Personen limitiert. Vier Mitglieder werden von den Mitarbeiter*innen gewählt, vier sind verdiente Fördermitglieder und weitere vier Mitglieder werden vom Vorstand vorgeschlagen. Die Mitgliederversammlung fungiert als Aufsichtsrat, trifft strategische Entscheidungen und wählt den geschäftsführenden Vorstand. Die Fördermitgliedschaft steht allen Einzelpersonen offen (Campact 2017). Campact definiert jede Person, die die Newsletter erhält, als Unterstützer*in. Die indirekte Erklärung für diesen Organisationsaufbau kann darin gesehen werden, dass Campact in seinem Selbstverständnis keine weitere NGO, sondern eine Plattform sein will (siehe Kapitel 4).

Im Zentrum des Organisationsgeschehens steht die Webseite als Beteiligungsinfrastruktur. Die Aktionsformen finden meist digital statt, bspw. in Form einer Petition, die an den Versand von standardisierten E-Mails an politische Entscheidungsträger*innen gekoppelt ist. Die Möglichkeiten der Beteiligung sind punktuell, allerdings wird eine dauerhafte Bindung der Unterstützer*innen an die Organisation über individualisierte Angebote forciert. Auch jenseits des digitalen Protestes steht es Interessierten offen, analog an Demonstrationen, Aktionen oder Veranstaltungen teilnehmen. 
Einzelpersonen können zudem die angegliederte Protestplattform „WeAct“ nutzen, um eigenständig Petitionen zu starten. Dort werden die technische Infrastruktur und andere Hilfestellungen zur Verfügung gestellt. Dem eigenen Selbstverständnis folgend, wird den Unterstützer*innen eine spezifische Option angeboten, zu einem bestimmten Thema tätig zu werden. Den verschiedenen Einschätzungen zufolge, funktioniert Campact ausdrücklich durch die Menschen, die sich mobilisieren lassen wollen. Dieser Sichtweise folgend, stellt nicht Campact die Forderungen an die Politik, sondern die jeweiligen Petitionsunterstützer*innen.

Die Mitgliederkommunikation verläuft professionell und zielgruppengerecht top-down. Campacts größter Asset sind die Newsletterabonnement*innen. Alle gängigen Social Media Kanäle werden voll ausgespielt, es ist sogar eine direkte Kommunikation über Messenger-Dienste möglich. Die Themenwahl findet in einer Kombination aus top-down Vorentscheidung und bottom-up Rückversicherung statt (siehe Infobox zu Campact, Abbildung 5.3).

Foodwatch ist ein eingetragener Verein, der als gemeinnützig anerkannt ist. Auch Foodwatch hat sich für eine abgestufte Mitgliederstruktur entschieden. Die Anzahl der Vollmitglieder ist satzungsgemäß auf 100 Mitglieder beschränkt; eine Fördermitgliedschaft steht jedem offen. Die Fördermitglieder sichern die finanzielle Unabhängigkeit der Organisation. Daher wird die dauerhafte Bindung der Fördermitglieder forciert. Allerdings ist der Aufbau dezentraler und ehrenamtlicher Strukturen nicht geplant. Die Wahl einer abgestuften Mitgliederstruktur wird mit der Sorge vor einer gezielten Unterwanderung erklärt, welche die Handlungsfähigkeit der Organisation blockieren könnte. Die Geschäftsstelle fungiert als Organisationsspitze.

Durch die fehlenden ehrenamtlichen Strukturen ist die Beteiligungsmöglichkeit der Fördermitglieder und Unterstützer*innen eingeschränkt. Dies wird mit der Sorge vor juristischen Konsequenzen bei ehrenamtlichem Engagement in speziellen und technisch anspruchsvollen Themen des Lebensmittelrechts gerechtfertigt. Es besteht allerdings die Möglichkeit, online Petitionen zu unterschreiben und an Mailingaktionen teilzunehmen. Auch das Melden von „Werbelügen“ im Lebensmittelbereich oder die Abstimmung für den jährlich vergebenen Negativpreis sind auf der Internetseite möglich.

In gleicher Weise können die Unterstützer*innen an Demonstrationen und Aktionen teilnehmen. Auch wenn die Beteiligungsoptionen punktuell möglich sind, wird eine kontinuierliche und feste Fördermitgliedschaft angestrebt. Daher gibt es auch spezielle Angebote und Informationen für diese Mitglieder. Die Kommunikation mit der Organisationsspitze findet hauptsächlich in den Social Media 


\section{Campacts Themenwahl und intelligente Internetseite}

Das Selbstverständnis von Campact als thematisch nicht festgelegte Protestplattform bzw. als Protestverstärker, findet Ausdruck in der Interaktion, Kommunikation und Bindung ihrer Unterstützer*innen. Campact führt auf seiner Internetseite an, knapp 2,2 Millionen Unterstützer*innen zu haben (Campact 2019c). Diese Anzahl leitet sich von den Newsletter-Abonnent*innen ab. Jede Abonnent*in ist eine Unterstützer*in. Diese Newsletter-Abonnent*innen sind Campacts größter Asset innerhalb der Plattforminfrastruktur, denn der Kontaktdatenpool konstituiert das Mobilisierungspotenzial.

Campact richtet seine Kampagnenaktivität an aktuellen politischen Chancen und Veränderungsmomenten aus. Die Vorauswahl der möglichen Kampagnenthemen erfolgt auf Grundlage spezieller Kriterien innerhalb der Geschäftsstelle. Zunächst muss ein Thema ein generelles Interesse in der Bevölkerung hervorrufen. Ein politischer Kristallisationspunkt bspw. eine anstehende politische Entscheidung muss greifbar sein. Diese Entscheidungssituation muss offen genug sein, sodass sie in die eine oder andere Richtung ausgehen kann. Zudem muss die politische Entscheidung gewinnbar sein, also mindestens begrenzte Aussicht auf Erfolg haben. Hierfür muss das Streitthema medial anschlussfähig (d.h. nicht zu komplex, dafür jedoch zuspitzbar) sowie kanalisierbar sein (Die Gesellschafter 2009b, Bautz 2008: 108-110).

Auf diese Weise identifiziert die Organisationen einen Pool an potenziellen Kampagnenthemen, die mit professionellen Instrumenten der Marktforschung getestet werden. Zunächst findet für ein potenzielles Thema eine randomisierte Interessenabfrage unter den Unterstützer*innen statt. Das potenzielle Kampagnenthema wird nur aufgenommen, wenn es ein bestimmtes Quorum an positivem Rücklauf erhält. Nachdem das Thema über diese Schwellen abgesichert ist, gibt es die Möglichkeit online an einer thematisch entsprechenden Petition teilzunehmen. Auf Grundlage der vergangenen Teilnahmen an Petitionen wird ein personalisiertes Benutzer*innenprofil erstellt. Für die individuelle Nutzer*in kann auf diese Weise ein maßgeschneidertes Angebot an Themen, Veranstaltungen und Aktionen generiert werden, um die persönlichen Präferenzen effizient anzusprechen.

Ein Kernstück des strategischen Aufbaus von Campact liegt in der technischen Umsetzung der Infrastruktur ihrer Webseite - ein innovatives Instrument der zivilgesellschaftlichen Mobilisierungsarbeit.

\section{Abbildung 5.3 Infobox zu Campact}


Communities statt. Dort wird ein dialogischer Austausch verfolgt. Weitere Kommunikationskanäle sind E-Mails oder auch eine telefonische Verbraucherhotline, die stundenweise aktiv ist.

Die Themenwahl wird zentral in der Geschäftsstelle vorgenommen. Neben einer groben thematischen Jahresplanung wird flexibel auf aktuelle Skandale oder aufgedeckte Missstände reagiert. Themenvorschläge werden auch über die Kommunikationskanäle der Organisation eingebracht. Am Ende trifft allerdings die Geschäftsstelle die Entscheidung.

Die Deutsche Umweltstiftung ist als gemeinnützig anerkannte, rechtsfähige Stiftung bürgerlichen Rechts - Bürgerstiftung - gegründet. Ihre aktuelle Stifter*innenanzahl wird auf der Internetseite der Umweltstiftung bekanntgegeben. Die Konstitution als Stiftung stellt andere Voraussetzungen an den organisationalen Aufbau und die Funktionsorgane. So gibt es einen geschäftsführenden Vorstand und ein prüfendes Kuratorium. Satzungsgarantierte Rechte oder Pflichten kommen den einzelnen Stifter*innen nicht zu.

Im Stiftungszweck verankert ist das Ziel, zur Bildung und Informationen über Umweltschutzthemen beizutragen und Dritte finanziell bei diesen Aufgaben zu unterstützen. Das Bildungsangebot richtet sich auch an die Stifter*innen. Mit der neuen Akzentuierung auf Bürgerbeteiligungsformate und dem Aufbau des „Berlin Instituts für Partizipation" rücken diese Themen in den Fokus. Durch die Crowdfunding Plattform „Ecocrowd“ können Interessierte selbst für eigene Anliegen werben - dem Stiftungsmotto „Hoffnung durch Handeln“ folgend.

Öffentliche Kommunikation findet über Newsletter und Social Media, hier vor allem Facebook, statt. Ob es weitere Stifter*innen-interne Kommunikationsformate gibt, kann aufgrund des begrenzten Datenmaterials nicht näher expliziert werden. Die Internetseite als Informationsquelle ist sehr statisch aufgebaut. Unter dem Reiter ,Aktuelles' finden sich Hinweise zu Aktionen. Im Rahmen der Recherchen konnten keine Informationen über Rückkopplungsmöglichkeiten, Interaktion oder Inputmöglichkeiten zwischen dem geschäftsführenden Vorstand, Mitarbeiter*innen und Stifter*innen offengelegt werden.

\subsection{Auswertung der theoriegeleiteten Kategorie der Einflusslogik}

Das theoriegeleitete Kategorienset der Einflusslogik umfasst u. a. die Kategorien Instrumenteneinsatz, ,inside lobbying “ über Fachexpertise oder persönliche Kontakte, „outside lobbying“ über öffentliche Sichtbarkeit und politischen Druck sowie Bündnisse und Kooperationen (Abbildung 5.4). 


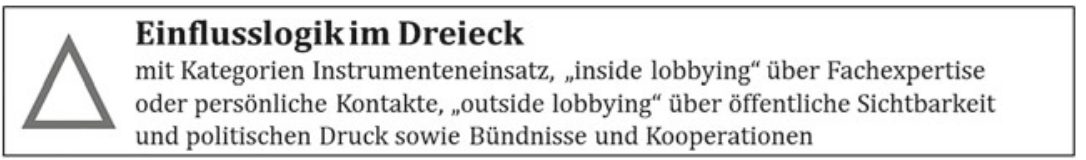

(Grafik: eigene Darstellung)

Abbildung 5.4 Hauptkategorie der Einflusslogik

Attac ist dem eigenen Selbstverständnis folgend zwischen den idealtypischen Organisationsformen NGO, Netzwerk und Bewegung verortet. Der strategische Schwerpunkt der Organisation liegt im Bereich der Basisarbeit und der Aktions- und Kampagnenorganisation (siehe Infobox zu Attac und Abschnitt 5.4). Die Professionalisierung der politischen Interessenvermittlung zeigt sich bei Attac an einem breiten Instrumenteneinsatz der politischen Einflussnahme. Der systematischen Aufteilung folgend, dominiert Outside-Lobbying deutlich über Inside-Strategien. Im Outside-Bereich überwiegen Bildungsarbeit sowie der Expertise-Aufbau auf Seiten der Aktiven. Diese Bildungsarbeit richtet sich an alle Interessierten und Unterstützer*innen. Als Instrument des indirekten politischen Einflusses zielt sie darauf ab, Einstellungen und Problembewusstsein zu verändern. Dies ist für die Organisation der bevorzugte, aber langwierige Weg. Der Inside-Bereich erfolgt über die Ansprache von politischen Entscheidungsträger*innen und über das Angebot zur Bereitstellung von Expertise zu den Kernthemen der Organisation. Allerdings ist es laut Interviewaussagen nicht die Hauptstrategie, „klassischen Lobbyismus“ zu betreiben.

Für die Fachexpertise kann Attac auf einen wissenschaftlichen Beirat zurückgreifen, der u. a. bei Fachthemen hilft, Informationsmaterialien zu erstellen. Der Kontakt zu Abgeordneten und Entscheidungsträger*innen erfolgt meist öffentlich bei Veranstaltungen oder im Vorfeld von wichtigen Entscheidungen zu Finanzmarktthemen. Kontakte und Netzwerke bestehen u. a. über Personen, die nach ihrer Tätigkeit bei Attac parteipolitische Ämter erlangten. Auf diese Weise bestehen Kontaktwege in die Politik hinein. Prominentes Beispiel ist der Europaabgeordnete der Grünen, Sven Giegold.

Im Bereich der öffentlichen Sichtbarkeit innerhalb der Einflusslogik sind die Medienarbeit (siehe Abschnitt 5.3) sowie die Ansprache von politischen Entscheidungsträger*innen im Rahmen von Kampagnen zu nennen. Hierfür verfügt die Organisation über ein Kampagnenteam, welches öffentlichkeitswirksame Aktionen und Protestformen plant und durchführt. Beispielhaft war hier die Besetzung 
der Paulskirche im Herbst 2018, um an den 10. Jahrestag der Finanzkrise zu erinnern. Vielfach werden Kunstaktionen und kreative Aufführungen eingesetzt, um die mediale Sichtbarkeit und die Aufmerksamkeit beim Unterstützer*innenkreis zu erhöhen.

Das Selbstverständnis als Netzwerk zeigt sich daran, dass zahlreiche Organisationen - u. a. aus dem Bereich Gewerkschaften, Kirchen und Entwicklungszusammenarbeit - Mitglied bei Attac sind. Dies erleichtert thematische Kooperationen bzw. Themenallianzen mit zivilgesellschaftlichen Partnern. In den Bereich der Kooperation fällt zudem, dass für gemeinsame Projekte teilweise Drittmittel akquiriert werden können. Kritisch wurde von den Interviewpartner*innen angemerkt, dass die Teilnahme an großen Bündnissen dazu führen kann, dass das Engagement von Attac nicht ausreichend registriert wird (siehe Grenzen der politischen Wirksamkeit, Abschnitt 5.4). So wurde Attac bei der Anti-TTIP/CETA-Kampagne weniger stark als erhofft wahrgenommen, obwohl mit Finanzmarkt- und Welthandelsthemen die Kernanliegen der Organisation betroffen waren.

LobbyControl richtet den Fokus auf die beiden Säulen der Kampagnenund der Expertisearbeit. Dies zeigt sich auch beim Instrumenteneinsatz, der sich stark an der eigenen Fachexpertise, Professionalisierungsanforderungen und dem Schwerpunkt der Expertisegenerierung ausrichtet. Hierzu zählen Medienarbeit, öffentlichkeitswirksame Aktionen und Kampagnen, Stellungnahmen in Parlamenten, Netzwerkarbeit und Themenbündnisse mit Partnerorganisationen, Online-Petitionen sowie Hintergrundinformationen zu den Positionen der Parteien zu Lobbyregulierung.

Der Bereich des Inside-Lobbying steht im Zeichen dieser Expertise-Strategie. Da die Themen der Lobbyregulierung komplex sind, benötigt die Organisation eine hohe Fachkenntnis, um Problemanalysen, Handlungsoptionen und Lösungsvorschläge liefern zu können. Diese Fachexpertise richtet sich häufig direkt an politische Entscheidungsträger*innen. Teilweise geschieht das über Hintergrundgespräche, offene Briefe sowie über zahlreiche Parlamentsanhörungen auf Bundes- und Länderebene, an denen LobbyControl mit Sachverständigen teilnimmt. Diese Ausrichtung folgt der Einschätzung, dass eine Organisation ein Alleinstellungsmerkmal ausarbeiten muss, um für politische Ansprechpartner*innen interessant zu sein.

Auch im Outside-Bereich kombiniert LobbyControl die beiden Säulen, um öffentliche Sichtbarkeit zu erlangen. LobbyControl dokumentiert bspw. die Fälle von Seitenwechsel aus der Politik in die Wirtschaft und bereitet sie in einer Datenbank auf. Oftmals führen prominente Fälle dazu, dass dieser Wechsel medial 
beachtet wird und teilweise in die Skandalisierungslogik in der Medienberichterstattung fällt. Dies versucht die Organisation zu nutzen, um den Umfang und die Relevanz des Themas aufzuzeigen und um Druck auf politische Entscheidungsträger*innen aufzubauen. Als ausschlaggebendes Kriterium wird hier die mediale Stellung als Expertenorganisation gesehen, um aktiv von Medien angefragt zu werden. Daneben stellt LobbyControl eigene Rechercheergebnisse sowie wissenschaftliche Erkenntnisse und Studien zusammen. Als Mittel der öffentlichen Interessenvertretung werden Interviews und Stellungnahmen in der medialen Berichterstattung zu politischen Skandalen sowie eigene öffentliche Events genutzt, um die eigenen Forderungen u. a. nach Karenzzeiten, Lobbyregistern und anderen Kampagnenthemen zu verbreiten.

Bedeutend aus der Perspektive der Einflusslogik ist die kontinuierliche Netzwerkarbeit mit Verbündeten auf Seiten der Politik und der Zivilgesellschaft. LobbyControl setzt auf ein Netzwerk von politisch relevanten Akteuren in Berlin und Brüssel. Zudem werden persönliche Kontakte aufgebaut, bspw. indem einzelne Abgeordnete direkt angesprochen werden. Allerdings geht die Organisation keine direkten Allianzen mit Politiker*innen ein, da ihr Distanz und Unabhängigkeit wichtig sind.

Wenn Themenallianzen gebildet werden, prüft die Organisation die möglichen Partner. Denn die eigene Glaubwürdigkeit wird als höchstes Gut angesehen, um in den politischen Prozessen wirken zu können. Dies folgt auch hier der Einschätzung, dass zivilgesellschaftliche Organisationen besser gehört werden, wenn sie große Bündnisse schmieden. Besonders deutlich zeigt sich die Kombination aus Expertise und Kooperation am Gesetzentwurf für ein verpflichtendes Lobbyregister, das die Organisation gemeinsam mit der NGO Abgeordnetenwatch erstellte. In einem längeren Prozess mit Onlinebeteiligung wurden die Zwischenergebnisse auf einer Webseite veröffentlicht und zur Kommentierung freigegeben. Gemeinsam präsentierten die Organisationen eine überarbeitete Version des Entwurfs bei einer Pressekonferenz Anfang 2017 im Vorfeld der Bundestagswahl. Ziel war es, die stillstehende politische Debatte über das Thema mit einem konkreten Vorschlag zu beleben (LobbyControl 2017a: 6, 2017b: 12-16; siehe Schiffers 2018a) (Abbildung 5.5).

Campact versteht sich explizit nicht als weitere inhaltlich orientierte FachNGO, sondern in der Rolle einer Beteiligungsplattform als schlagfertige Ergänzung im breiten Organisationsfeld der Zivilgesellschaft. Die Organisation zielt darauf ab, eine große Zahl an Unterstützer*innen punktuell zu mobilisieren und politische Entscheidungen über Petitionen zu beeinflussen. Dies zeigt sich auch an ihrem Instrumenteneinsatz. Als Plattform, um Bürgerprotest zu organisieren, übernimmt die Webseiten-Infrastruktur eine zentrale Stellung. Auf diese Weise 


\section{LobbyControl - Watchdog mit langem Atem}

Seit der Gründung von LobbyControl im Jahr 2005 ist die Einführung eines verpflichtenden Transparenz- und Lobbyregisters für Deutschland ein zentrales Anliegen der Organisation. Um dieses Ziel zu erreichen, kombiniert LobbyControl punktuelle und kontinuierliche Themenbearbeitung. Das setzt zum einen konstantes, kritisches und investigatives Arbeiten sowie zum anderen ausgeprägtes Durchhaltevermögen und einen langen Atem voraus. Durch diese Art der dauerhaften, wachsamen Auseinandersetzung kann LobbyControl das Attribut einer watchdogOrganisation zugeschrieben werden. Die zivilgesellschaftliche watchdog-Funktion bezieht sich darauf, u.a. politische, gesellschaftliche und wirtschaftliche Missstände aufzudecken. Im Sinne einer sozialen Regulierung dient die Praxis des „naming and shaming“ (Beer/Bartley/Roberts 2012) einer kritischen Öffentlichkeit.

Bei LobbyControl zeigt sich deutlich die doppelte Ausprägung des Agenda Setting in der watchdog-Strategie von NGOs. Die zivilgesellschaftliche Wächterfunktion teilt sich zum einen in die Strategie auf, punktuelle Impulse und Interpretationsangebote in den Aufmerksamkeitszyklen der medialen Debatte zu setzen. Zum anderen umfasst sie die Strategie eines kontinuierlichen Auf-der-Agenda-haltens, um die eigenen Positionen zu re-thematisieren und um einen Politikwandel anzustreben (Schiffers 2018a).

\section{Abbildung 5.5 Infobox zu LobbyControl}

kann Campact enorm schnell agieren, um ein hohes Maß an Mobilisierung und politischer Lautstärke zu generieren. Inhaltlich richtet sich diese Mobilisierungsarbeit an speziell ausgewählte, kriterienbasierte Themen (siehe Infobox zu Campact in Abschnitt 5.1), bei denen politische Entscheidungen auf der Kippe stehen und mittels der Petitionen Erfolge erzielt werden können. Dabei hat die Organisation keine thematischen Einschränkungen, sondern folgt den eigenen, normativ übergeordneten politischen Zielen des sozialen, ökologischen und demokratischen Fortschritts. Diesem Zuschnitt folgend dominiert fast ausschließlich die OutsideStrategie. Über öffentliche Interessenvertretung als Verstärker für Protest soll politischer Druck aufgebaut werden.

Die Organisation übernimmt die Rolle als Mobilisierungsagentur bzw. als Vermittler und Verstärker zwischen zivilgesellschaftlichen Anliegen und dem 
Personenkreis der Unterstützer*innen. Für Campact steht das professionalisierte Wissen zu Campaigning, Mobilisierung und Kommunikation im Vordergrund. Abweichend von Verbänden oder anderen NGOs gibt es keine thematischen Fach-Referent*innen, sondern Campaigner*innen mit ausgeprägter Arbeitserfahrung in Schwerpunktthemen, die einzelnen Kampagnen zugeordnet werden (Speth/Zimmer 2015). Daher unterscheidet sich auch das Grundverständnis der Organisation. Klassisches Inside-Lobbying über Expertise oder direkte Kontakte wird nicht verfolgt. Politische Entscheidungsträger*innen werden lediglich über Mailingaktionen oder über medial sichtbare Protestaktionen angesprochen. Der Strategiezuschnitt ist darauf ausgelegt, Politiker*innen über Veränderungen in der öffentlichen Meinungs- und Stimmungslage zu erreichen. Öffentliche Sichtbarkeit und Unterstützung sind daher ein wichtiges Mittel.

Der strategische Weg von Campact verläuft vielmehr über Partnerorganisationen, die die fachliche Expertise sowie teilweise die Gesichter für Kampagnen liefern und welche über die professionalisierte Kampagneninfrastruktur organisiert werden. Bündnisse und Kooperation übernehmen daher eine entscheidende Rolle im Arbeitsselbstverständnis.

Foodwatch orientiert seine organisationale Grundausrichtung am Selbstverständnis einer politischen Kampagnenorganisation. Auch bei der Instrumentenwahl stehen Medien- und Kampagnenarbeit im Vordergrund. Das Ziel ist Agenda-Setting und Problembenennung zu den Kernthemen, für welche darüber hinaus auch Lösungsangebote präsentiert werden. Die Strategie, die hierfür genutzt wird, basiert auf einer konfrontativen Grundhaltung (siehe Infobox zu Foodwatch in Abschnitt 5.3).

Im Inside-Bereich - und trotz der grundlegenden Orientierung auf Konfrontation - spielt Fachexpertise eine große Rolle. Die Organisation produziert umfassende Reports als Basis ihrer Recherche- und Informationsarbeit, um ihre Glaubwürdigkeit und Seriosität in der breiten medialen Öffentlichkeit und in der Fachdebatte zu untermauern. Allerdings unterscheidet sich die Intention der Strategie: Expertisen und Reports dienen insbesondere als Kommunikationsanlass. Sie bilden die Grundlage für die darauffolgenden Kampagnen, in denen auch explizit konfrontative Taktiken gefahren werden. Die Organisation formuliert für diese strategische Charakteristik selbst den „Anspruch, die Dinge beim Namen zu nennen“, was sie ,allerdings nur nach gründlicher Recherche und Analyse" mache (Foodwatch 2019b). Klassisches Lobbying über persönliche Kontakte und Gremienteilnahme ist nach eigenen Aussagen nicht Kern der Strategie. Dennoch bestehen Gesprächsangebote an alle Seiten. Vertreter*innen von Foodwatch werden aber durchaus für die Rolle als Sachverständige und Expert*innen in Parlamentsausschüsse eingeladen und nehmen diese Angebote auch wahr. 
Die Ausrichtung auf Medien- und Kampagnenarbeit stellt den OutsideBereich mit professionalisierter Öffentlichkeitsarbeit in den Vordergrund (siehe Abschnitt 5.3). Foodwatch hat das Ziel, politische Veränderungen über öffentlichen Druck zu erreichen. Hierfür orientiert sich die Organisation an der idealtypischen Abfolge, Probleme aufzudecken, sie auf die politische Agenda zu bringen, Druck auszuüben und die Problemlösung kritisch zu begleiten. Die Mittel hierfür sind Pressekonferenzen, öffentlichkeitswirksame Aktionen, Petitionen und Mailaktionen an Abgeordnete, Mitmachoptionen auf der Webseite sowie zugespitzte Kritik. Zudem nutzt die Organisation auch juristische Wege, etwa über Verbandsklagen (als qualifizierte Einrichtung im Sinne des Unterlassungsklagengesetzes; Bundesamt für Justiz 2019).

Im Bereich der Netzwerkarbeit zeigt sich bei Foodwatch die Besonderheit, dass es mittlerweile einen internationalen Ableger der Organisation gibt. Als Dachorganisation verbindet Foodwatch International die nationalen Organisationen in Deutschland, Frankreich und den Niederlanden.

Die Deutsche Umweltstiftung legt den Schwerpunkt auf Projekte zur Bildungs- und Informationsarbeit. Politische Interessenvertretung über Inside und Outside-Strategien können auf Grundlage des begrenzten Datenmaterials nicht gesondert dargestellt werden. Die politische Wirkung erfolgt insbesondere indirekt über die Projekte. Über Information, Bildung und Aufklärung soll das Umweltbewusstsein vieler Menschen - gerade auch über den Stifter*innenkreis hinaus - verändert werden. Die Organisation setzt laut eigenen Aussagen auf „Eigenverantwortung statt Bevormundung“ sowie auf ,private Initiative statt staatlicher Bürokratie“" (LifeVerde 2019). Organisational wird diese Grundorientierung durch einen Wirtschaftsrat unterstützt. Darin versammeln sich Unternehmensvertreter*innen verschiedener Branchen, die sich im Bereich Nachhaltigkeit engagieren. Sie teilen sich die Gemeinsamkeit, dass ihr Kerngeschäft bereits nachhaltig aufgestellt ist oder daran gearbeitet wird (Deutsche Umweltstiftung 2015: 8). Auch hier wird versucht, die übergeordneten politischen Ziele über freiwillige Selbstverpflichtung mit der Wirtschaft umzusetzen.

Weitere Instrumente der politischen Arbeit sind die Durchführung von Tagungen und Veranstaltungen (Deutsche Umweltstiftung 2015, 2012) sowie die Mitfinanzierung von Publikationen, Ausstellungen, Fotobänden und Wettbewerben zu Umweltthemen (Infobroschüre 2012; aktuellere Informationen liegen nicht vor). Zentrale Rolle im Schwerpunkt der Projektarbeit übernehmen die Ausgründungen der Crowdfunding Plattform „Ecocrowd“ sowie des „Berlin Institut für Partizipation" bipar (siehe Kapitel 4). Sowohl in Kooperation mit Partnerorganisationen als auch eigenständig initiierte die Umweltstiftung zahlreiche Projekte (Deutsche Umweltstiftung 2012: 3). Hierunter fallen Baumpflanzaktionen und die 
Unterstützung lokaler Naturinitiativen (LifeVerde 2019; Deutsche Umweltstiftung 2011 [1982]).

\subsection{Auswertung der theoriegeleiteten Kategorie der Reputationslogik}

Die Reputationslogik gliedert sich u. a. in die Kategorien öffentliche Anerkennung der Expertise, mediale Anerkennung des Akteurs und Themas sowie Elemente der professionalisierten Medienarbeit (Abbildung 5.6).

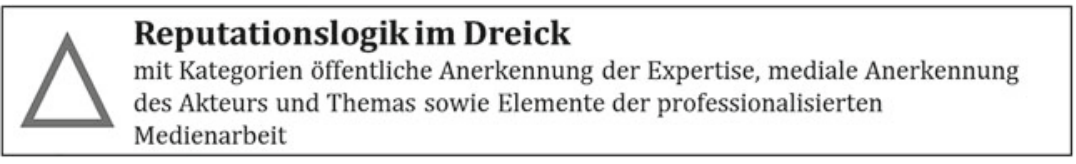

(Grafik: eigene Darstellung)

Abbildung 5.6 Hauptkategorie der Reputationslogik

Attac betreibt eine professionelle Presse- und Öffentlichkeitsarbeit. $\mathrm{Zu}$ den Instrumenten zählen inhaltlich zugespitzte Pressemitteilungen und -erklärungen sowie die gezielte Einladung zu Pressekonferenzen. Die Organisation hat laut Interviewaussagen kontinuierliche, persönliche Beziehungen zu Journalisten*innen und Medien, die den Themen der Organisation nahestehen und Attac häufig als Gesprächspartner wählen.

In den Kernthemen (Finanztransaktionssteuer, Globalisierungskritik) wird Attac als kompetenter, glaubwürdiger Akteur anerkannt und häufig als kritischer Vertreter der Gegenposition gegenüber Parteipolitiker*innen angefragt. Attac sieht sich den Zwängen der Medienlogik ausgesetzt und nimmt die Konkurrenz um Aufmerksamkeit und die Spielarten medialer Themenkonjunkturen deutlich wahr. Dies ist laut Interviewaussagen eng gekoppelt mit der Erfahrung aus der Zeit der Finanzkrise und den Anti-TTIP/CETA-Demonstrationen. Obwohl dort die Attac-Themen krisenbedingt Konjunktur hatten und die Organisation als Expertin angefragt wurden, konnte das Engagement nur begrenzt politische Veränderung bewirken. 
Im Rahmen der Öffentlichkeitsarbeit ist der Organisation bewusst, welche Themen medial gut aufgenommen werden: emotionale Themen, Skandale und wenig komplexe Inhalte. Die Themenauswahl werde daran allerdings nicht ausgerichtet. Stattdessen versucht Attac, die eigenen, als relevant identifizierten Themen medial aufzubereiten, etwa über künstlerische Aktionen.

LobbyControl zeichnet sich ebenfalls durch professionelle Presse- und Öffentlichkeitsarbeit aus und verfügt über ein gewachsenes Kontaktnetzwerk von Journalist*innen. Aufgrund der organisationsintern etablierten Fachexpertise wird LobbyControl als glaubwürdiger, seriöser und relevanter Akteur anerkannt und von Medien als Gesprächspartner zu den Kernthemen angefragt. Neben der wachsenden Bereitstellungsfunktion von Hintergrundinformationen für Medien, wird LobbyControl häufig als Gegenstimme zu Politiker*innenaussagen medial inszeniert. Ein verantwortungsvoller Umgang mit dieser Rolle wird als zentral angesehen, um glaubwürdig zu bleiben. Dies stelle hohe Ansprüche an die eigene Expertenrolle. Laut den Interviewaussagen werden NGOs als ein relevanter Akteur unter vielen in der pluralen Medienlandschaft gesehen, welcher allerdings nicht besonders von der modernen Medienlogik profitiere.

Der eigenen Einschätzung nach besteht ein gutes Verhältnis zu den Medien und ein grundsätzliches Interesse an den bearbeiteten Themen. Viele davon sind allerdings sehr komplex, sodass sie medial weniger gut aufgearbeitet werden können. Daher besteht die Schwierigkeit, mit den eigenen Themen mediale Resonanz zu erfahren und nicht nur als Experte für verwandte, weniger komplexe Themen angefragt zu werden.

Campact nutzt ihre professionelle Presse- und Medienarbeit über das Versenden von Pressemitteilungen, Kontakte mit Journalist*innen und das Bereitstellen von Informationsmaterial zu Kampagnen und Aktionen. Die Pflege der Social Media Kanäle scheint für Campact allerdings deutlich wichtiger zu sein, als der klassische Medienkontakt. Dies zeigt sich an der personellen Aufteilung von zwei Mitarbeiter*innen in der Pressestelle und sechs Mitarbeiter*innen in der Social Media Abteilung (Campact 2019b). Die öffentliche Sichtbarkeit - nicht nur medial, sondern vor allem auf Social Media Plattformen - gilt als höchstes Gut. Kampagnenaktionen, Mobilisierung und öffentlich aufgebauter Druck verfangen nicht ohne mediale Begleitung. Campact übernimmt nicht die Rolle eines Fachexperten, sondern wird von den Medien eher als Lieferant guter Bilder gesehen. 
Aufgrund der Wahrnehmung, dass die persönliche Agenda der Menschen primär von Massenmedien bestimmt wird, befindet sich Campact kontinuierlich im medialen Austausch. Die Organisation nimmt Themen auf, welche politische Konjunktur und die Chance auf Erfolg haben. Bei der Themenwahl beschränkt sich Campact darauf, nur wenige Kampagnen gleichzeitig zu bearbeiten (siehe Infobox Campact, Abschnitt 5.1). Andernfalls drohe die Gefahr, in der Konkurrenz um Aufmerksamkeit mit wenig erfolgsversprechenden Themen unterzugehen.

Foodwatch pflegt ebenfalls eine professionelle Öffentlichkeits- und Pressearbeit. $\mathrm{Zu}$ den Instrumenten zählen Pressemitteilungen, Pressekonferenzen und persönliche Gespräche im Austausch mit Journalist*innen. Als Teil der Öffentlichkeitsarbeit werden die Social Media Kanäle professionell bespielt. Die Medienarbeit berücksichtigt die Anforderungen der unterschiedlichen Teilöffentlichkeiten mit einer differenzierten Ansprache der Unterstützer*innen, der breiten Gesellschaft, der politischen Entscheidungsträger*innen und des Fachpublikums. Insbesondere die Veröffentlichung von hausintern generierten Studien und Reports wird als Kommunikationsanlass genutzt. Darüber hinaus soll Foodwatch mit dem Aufbau von Fachexpertise als glaubwürdiger und seriöser Akteur wahrgenommen werden. In dieser Rolle wird die Organisation selektiv von Medien als Gesprächspartner kontaktiert.

Mit den bearbeiteten Themen zielt Foodwatch darauf, Agenda Setter zu sein. Trotzdem ist die Themenauswahl häufig kurzfristig und flexibel - sowohl bei Inhalten, die sich medienlogisch gut vermarkten lassen, als auch bei anderen, für die Organisation als relevant identifizierten Themen (Abbildung 5.7).

Die Deutsche Umweltstiftung betreibt eine klassische, aber eher verhaltende Öffentlichkeits- und Pressearbeit. Auf der Webseite werden regelmäßig Pressemitteilungen veröffentlicht. Jürgen Sommer wird als langjähriger Vorsitzender der Deutschen Umweltstiftung verschiedentlich sowohl in einschlägigen Fachmedien (bspw. Movum 2019) als auch in klassischen Medien (bspw. Süddeutsche Zeitung) als Interviewpartner zu Umwelt- und Nachhaltigkeitsthemen aber auch Bürgerbeteiligungsformaten angefragt. Aufgrund des begrenzten Datenmaterials ist nicht festzustellen, ob die Deutsche Umweltstiftung als Organisation die Reputation eines relevanten, wichtigen Ansprechpartners für die Medien hat oder diesen anstrebt. Ob Themenplatzierung und Agenda Setting funktionieren, kann aufgrund mangelnder Datenlage nicht abschließend beurteilt werden. 


\section{Foodwatch - die politische Kampagnenorganisation}

Foodwatch zeichnet sich in der Praxis und in der Selbstwahrnehmung durch einen deutlich konfrontativ ausgerichteten Kommunikationsstil aus. Ihre zugespitzten politischen Forderungen zu Themen im Lebensmittelsektor werden konfliktiv und lautstark in der Öffentlichkeit vertreten, um so Druck auf die Politik und betroffene Unternehmen ausüben zu können. Grundsätzlich ist die Organisation zwar für Gespräche und den Austausch mit allen Akteuren offen, aber die Grundstrategie verläuft entlang von öffentlich laut vorgetragener Kritik und umfangreichen Forderungen. Unternehmenspartnerschaften oder Kompromisse, die die Forderungen der Organisation nicht erfüllen, werden abgelehnt.

Diese konfrontative Strategie wird durch umfangreiche, inhaltliche Recherche und Analyse untermauert. Der Aufbau von fachlichem Wissen mit der Inside-LobbyingStrategiewahl ist nicht als Angebot von Informationen und Expertise an politische Entscheidungsträger*innen misszuverstehen. Dennoch nimmt die Organisation an Sachverständigenanhörungen in Parlamenten teil. Die Veröffentlichung von Reports und Analysen dient Foodwatch in erster Linie als Kommunikationsanlass und Grundlage für darauf aufbauende Kampagnen und weniger als eigenständiges Instrument.

Abbildung 5.7 Infobox zu Foodwatch

\subsection{Auswertung der materialbasierten Kategorie (1) übergeordnete Strategie}

Neben den drei theoriegeleiteten Kategorien der Unterstützungs-, Einfluss- und Reputationslogik, zielte der explorative Zuschnitt der Forschungsstrategie darauf ab, querliegende Bereiche mit materialbasierten Kategorien abzubilden. Das erste dieser vier Kategoriensets thematisiert Überlegungen zur übergeordneten Strategie von NGOs, die im Überschneidungsbereich des Dreiecks der Handlungsrationalität liegen. Das Set gliedert sich in die strategische Grundausrichtung, den organisationalen Zuschnitt, Organisationsrollen und Arbeitsteilung sowie die politische Wirkung mit kritischer Sicht auf die eigenen Grenzen. In einem Großteil 
der Interviewaussagen werden NGOs (im weitgefassten Begriffsverständnis und im weiteren Kontext der NGO-Landschaft) als Treiber gesellschaftlicher Veränderungsprozesse gesehen, auch gegen das Beharrungsvermögen der politischen Prozesse (Abbildung 5.8).

\begin{tabular}{|c|c|}
\hline & $\begin{array}{l}\text { Kategorienset der übergeordneten Strategie } \\
\text { mit Kategorien strategische Grundausrichtung, organisationaler Zuschnitt, } \\
\text { Organisationsrollen und Arbeitsteilung sowie politische Wirkung } \\
\text { mit kritischer Sicht auf die eigenen Grenzen }\end{array}$ \\
\hline Attac & $\begin{array}{l}\text { - Schwerpunkt Basisarbeit und Aktions- bzw. Kampagnenorganisation } \\
\text { basisdemokratische Strategie mit starkem Einbezug } \\
\text { der „Attacies“ (Mitglieder und Nicht-Mitglieder) } \\
\text { - normative Zielvorstellung, wie Welt und Gesellschaft verbessert werden } \\
\text { können (Bildungsarbeit) }\end{array}$ \\
\hline LobbyControl & $\begin{array}{l}\text { - Schwerpunkt Expertise und Kampagnenorganisation } \\
\text { - Expertise-Strategie als „insight lobbying“ analog zu inside/outside lobbying } \\
\text { normative Zielvorstellung (Unterrepräsentation von Interessen), } \\
\text { kritische Sicht auf eigene Grenzen }\end{array}$ \\
\hline Foodwatch & $\begin{array}{l}\text { - Schwerpunkt Kampagnenorganisation } \\
\text { - deutlich prägnante und konfrontative Strategie } \\
\text { - Hochprofessionalisierte Medienarbeit, } \\
\text { Expertise (Studien und Reports) als Kommunikationsanlass }\end{array}$ \\
\hline Campact & $\begin{array}{l}\text { - Schwerpunkt Mobilisierung, Kampagnenorganisation und -Infrastruktur } \\
\text { - Mobilisierung über eigene Plattform ermöglicht kurzfristige, } \\
\text { zielgruppengerechte/individuelle und punktuelle Partizipationsmöglichkeit } \\
\text { - Keine eigene thematische Expertise, stattdessen Verstärker und } \\
\text { Arbeitsteilung }\end{array}$ \\
\hline $\begin{array}{l}\text { Deutsche } \\
\text { Umweltstiftung }\end{array}$ & $\begin{array}{l}\text { - Schwerpunkt Projektarbeit mit begleitenden Kampagnen } \\
\text { - Bürgerstifter*innen im Mittelpunkt: Bildungs- und Informationsarbeit } \\
\text { - Wechselnde Kerngebiete: Fundraising-Plattform, Beteiligungsinstitut }\end{array}$ \\
\hline
\end{tabular}

(Grafik: eigene Darstellung)

Abbildung 5.8 Übersicht der NGO-Profile im Kategorienset der übergeordneten Strategie

\section{Strategische Grundausrichtung}

Attac akzentuiert die drei Säulen Expertise, Aktion und Bildung. Für die Organisation liegt der Umsetzungsmechanismus im Bewusstsein der eigenen Engagementmöglichkeiten und im Wissen über alternative Gestaltungswege bei (finanz-) wirtschaftlichen Themen. Hintergrund ist die normativ-idealistische Vision, gemeinsam eine gerechtere Gesellschaft erreichen zu können. Bildungsarbeit übernimmt darin die Rolle, ein grundlegendes Bewusstsein über die wirtschaftlichen Strukturen bspw. finanzwirtschaftlichen Themen zu schaffen, damit die 
komplexe Problemwahrnehmung ermöglicht wird. Die Säule der Aktion steht für die Mobilisierung der Aktiven und für das Ideal möglichst niedriger Beteiligungsschwellen, um sich mit eigenen Ideen einbringen zu können. Die Säule Expertise wird gespeist aus dem professionalisierten Arbeitswissen der Hauptamtlichen, über den wissenschaftlichen Beirat und aus dem Engagement der Aktiven. In organisationaler Hinsicht versteht sich die Geschäftsstelle als Dienstleisterin der Basis. Demnach kristallisiert sich als ein Schwerpunkt auf Basisarbeit und auf Aktions- bzw. Kampagnenorganisation als übergeordnete Strategie heraus.

Für LobbyControl mit den Säulen der Kampagnen- und der Expertisearbeit leitet sich der Handlungsimpuls aus dem Wissen über unterrepräsentierte Interessen und systematische Asymmetrien ab, die zivilgesellschaftlich gestärkt werden sollen. Die Zielsetzung nimmt Bezug auf demokratietheoretische Überlegungen, dass fairer Interessenausgleich durch verdeckte Einflussnahme erschwert wird. Dies gilt sowohl für einzelne Entscheidungen als auch für die Entscheidungsspielräume der repräsentativen Demokratie, wenn bestimmte, wirtschaftlich-starke Interessen systematisch bevorzugt werden. Für das Funktionieren der pluralistischen Demokratie ist nach dieser Auffassung eine starke Zivilgesellschaft notwendig. Mit der besonderen Rolle, die Fachwissen für die politische Arbeit einnimmt, lässt sich für LobbyControl eine Expertise-Strategie (,insight strategy“, Schiffers 2018) ausmachen, die Elemente der direkten und der indirekten Strategietypen kombiniert (analog zum inside und outside lobbying). Die übergeordnete Strategie legt den Schwerpunkt auf Expertise und Kampagnenorganisation.

Foodwatch sieht sich ausdrücklich als politische Kampagnenorganisation, um die Sichtbarkeit von Verbraucherrechtsthemen zu erhöhen und um Verbesserungen bei diesen Themen zu erreichen. Übergeordnet ist die Vorstellung, dass die Verbraucher*innen gegenüber der Lebensmittelindustrie aufgrund der informationellen Schieflage und dem Wunsch nach mehr Transparenz gestärkt werden müssen. Aus strategischer Sicht möchte die Organisation öffentlichen Druck auf Entscheidungsträger*innen und auf den Lebensmittelmarkt erzeugen, indem sie Missstände recherchiert, über verbrauchertäuschende Irreführung aufklärt und die Unterstützer*innen mobilisiert (Foodwatch 2019b, Die Gesellschafter 2009a). Die Organisation sieht eine Lücke in der politischen Einflussnahme zwischen Lebensmittelindustrie und Verbraucher*innen, die sie schließen möchte (Die Gesellschafter 2009a). Die strategische Grundausrichtung folgt dem Schwerpunkt der Medienarbeit und Kampagnenorganisation.

Campact versteht sich in der Rolle einer Beteiligungs- und Mobilisierungsplattform als Ergänzung zu Fach-NGOs. Campact wird aktiv, um Druck zu organisieren, wenn konkrete politische Entscheidungen anstehen bzw. auf der Kippe stehen und zugunsten wirtschaftlicher Partikularinteressen ausfallen können. An 
dieser Stelle sollen die Unterstützer*innen für Themen wie Menschenrechte, Umwelt- und Verbraucherschutz mobilisiert werden, um dazu beizutragen, den strukturellen Nachteil zwischen starken und schwachen Interessen auszugleichen (Speth/Zimmer 2015). Dem Organisationsverständnis folgend, werden die politischen Forderungen von den Unterstützer*innen und ihrem Engagement getragen. Die Organisation sieht sich als „Sprachrohr“ und als „Engagement-Netzwerk“ (Speth/Zimmer 2015). Der Schwerpunkt liegt damit auf der Mobilisierung und der Neugestaltung von Bürger*innenprotest, insbesondere durch die bereitgestellte digitale Infrastruktur.

Die Deutsche Umweltstiftung leitet ihr Selbstverständnis mit dem Stiftungsmotto „Hoffnung durch Handeln“ aus dem bürgerschaftlichen Engagement der Umweltbewegung der 1980er Jahre ab. Diese Maxime möchte gesellschaftliche Veränderungen durch individuelle Verhaltensänderungen erzielen: Die Bürgerschaft handelt selbst und wartet nicht auf politische Entscheidungen. Deshalb setzt die Stiftung nur indirekt auf die Veränderung von Politik; im Zentrum steht zivilgesellschaftliches Handeln. Laut dem begrenzten Datenmaterial und den Interviewaussagen verfolgt sie das Ziel, Umweltschutz durch eigene Informationsbzw. Bildungsformate sowie durch die ideelle oder materielle Unterstützung von Umweltprojekten Dritter zu fördern. Die umweltbewegten Überzeugungen sollen vernetzt in Gesellschaft und Politik wirken. Der Schwerpunkt liegt auf der Projektarbeit, die durch Kampagnen begleitet wird. Für die Zielsetzung der Bildungs- und Informationsarbeit stehen die Bürgerstifter*innen im Mittelpunkt. Bei den Projekten sind die Crowdfunding-Plattform „EcoCrowd“ und das Beteiligungsinstitut ,bipar“ zu nennen.

Über die oben dargestellten Strategie-Profile hinaus lassen sich in der qualitativen Analyse weitere Erkenntnisse herausarbeiten. Übergeordnet ist die Einschätzung, dass sich zivilgesellschaftliche Organisationen einem Kanon verschiedener Prinzipien verpflichtet sehen - wie Mitgliedern, Zielgruppen, Spender*innen, aber auch spezifischen Organisationskulturen und Werten. Hier schlagen sich auch Unterschiede in Größe und Ressourcenausstattung nieder, die zwischen den wenigen großen, meist internationalen NGOs und den vielen kleineren Organisationen differenzieren (Interview 21, 23). Dies resultiert in einer Herausforderung durch den Spagat zwischen den Anforderungen der Arbeitslogiken Kampagne, Expertise und Mitgliederkommunikation, die es notwendig macht, entweder Abstriche zu machen oder zusätzliche Stellen zu schaffen (Interview 30). 
Organisationaler Zuschnitt: Größe und Geschwindigkeit

In die Überlegungen zur strategischen Ausrichtung fließen Aspekte des jeweiligen organisationalen Zuschnitts von NGOs ein. Dies macht sich an den internen Strukturen, der Größe und an der Entscheidungs- und Handlungsgeschwindigkeit fest. Idealtypisch zeigt sich die Unterscheidung zwischen schnell handlungsfähigen, flexiblen und top-down organisierten NGOs einerseits und vielschichtig aufgestellten, basisdemokratischen, interessenaggregierenden und verbandsstrukturierten NGOs andererseits.

Organisationsstrukturell zeichnet sich der erste Typ durch kürzere Entscheidungswege, schnellere interne Abstimmungsprozesse und die Flexibilität aus, zügig auf aktuelle Anliegen zu reagieren. Der interne Ablauf stellt die Kampagnen als Produkt ins Zentrum. Sie bündeln die verschiedenen Aufgaben des Campaigning, der Medienarbeit, der Recherche, der Aktionsunterstützung, der Mitgliederarbeit und der Verwaltung (Die Gesellschafter 2009b, Speth/Zimmer 2015). Dieser Typ setzt weniger stark auf stimmberechtigte Mitgliedschaft, sondern auf Fördermitgliedschaften und die Mobilisierung für bestimmte Themen (Interview 19, 20). Das Engagement der Unterstützer*innen kann daher sehr punktuell ausfallen (Interview 23).

Beim verbandlichen, mitgliederbasierten Aufbau des zweiten Typs sind für Positions- und Entscheidungsfindung oder Neupositionierungen verhältnismäßig lange Zeiträume notwendig. Grund dafür ist, dass die internen Arbeitskreise, die Freiwilligenstrukturen und andere interne Gruppen aktiv mit einbezogen werden müssen. Zentrale Richtungsentscheidungen werden hier auf den meist jährlich stattfindenden Mitgliederversammlungen getroffen. Der vielschichtige Aufbau erlaubt zudem ein hohes $\mathrm{Ma} ß$ an Engagement von Engagierten und Aktiven der Basis im Sinne einer Mitmachorganisation (Interview 31, 16, 20, 23).

In der direkten Gegenüberstellung ist das höchste Pfund der jeweiligen Organisation entweder Flexibilität mit Handlungs- und Schlagfertigkeit oder Offenheit mit breiter demokratischer Absicherung nach innen (Die Gesellschafter 2009a, Interview 11, 26). Interviewaussagen deuten darauf hin, dass sich die lebhaft geführte Debatte über das eigene Idealprofil innerhalb zahlreicher NGOs zwischen diesen beiden Idealtypen orientiert (Interview 19, 21, 22, 18).

\section{Organisationsrollen und Arbeitsteilung}

Die Überlegungen zur übergeordneten Strategie schlagen sich zudem in den Organisationsrollen nieder, die einzelne NGOs in der Landschaft der Zivilgesellschaft übernehmen. Aus den Interviewaussagen lässt sich eine zivilgesellschaftliche Arbeitsteilung rekonstruieren. Die Bereitstellung der notwendigen Infrastruktur 
für Bündnisse, Gemeinschaftskampagnen, Demos, Großaktionen etc. ist nur möglich, wenn sich mehrere Organisationen zusammenfinden, um die Arbeiten und Kosten untereinander aufzuteilen (Interview 6, 30, 14). Die erkennbaren organisationalen Unterschiede im NGO-Mix können so im Sinne einer (bewussten oder unbewussten) Arbeitsteilung genutzt werden, um die jeweiligen Stärken situationsbedingt einzusetzen. Einzelne Organisationen können hier zum gemeinsamen Erfolg beitragen, wenn sie ihre Reaktionsschnelligkeit, ihre zugespitzte bzw. breitere Themenansprache, ihre Kampagnen-, Politik- und Expertisearbeit oder ihre Haltung für bzw. gegen Unternehmenskooperationen einbringen. Gleiches gilt für die verschiedenen Ansatzpunkte im Politikgestaltungsprozess. Politische Einflussnahme wird ausdifferenziert in allgemeine Bildungsarbeit sowie in die Artikulation, Mobilisation, Vernetzung und in klassische Lobbyarbeit (Interview 21, 17, 10). Metaphorisch kann dies damit verdeutlicht werden, dass einzelne Organisationen auf der Klaviatur der Zivilgesellschaft verschiedene Tasten drücken (Interview 21).

In dieser Gesamtkonstellation besteht die Anforderung, eine ökologische Nische für das eigene Profil in der Interessenlandschaft zu finden. Hierzu muss eine Organisation, so die Wahrnehmung vieler Akteure, ein eigenes strategisches Profil als Alleinstellungsmerkmal entwickeln und sichtbar machen. Denn die verschiedenen Organisationen stehen in der zivilgesellschaftlichen Konkurrenz einer pluralistischen Gesellschaft um Aufmerksamkeit und Ressourcen (Interview 20, 5, 14, 10, 6, 30, 2, Speth/Zimmer 2015).

Trotz der grundsätzlichen Kooperations- und Netzwerkorientierung müssen die Organisationen deshalb zwischen Arbeitsteilung - in Netzwerken und bei Themenkooperation - und Konkurrenz abwägen. Andernfalls droht laut einer Einschätzung die Gefahr, dass die Sichtbarkeit der einen Organisation bei einer gemeinsamen Aktion leidet, wenn sie hinter einer anderen Organisation zurückfällt oder in der Menge - z. B. bei der Anti-TTIP-Kampagne - untergeht. Dies hätte zur Folge, dass eine Organisation inhaltliches Engagement und Ressourcen investiert, ohne Mitgliedsschafts und Spendenzuwächse zu generieren.

Die zivilgesellschaftliche Arbeitsteilung hat eine weitere Grenze. Trotz der Professionalisierung der NGOs und ihrer Expertisegenerierung, bleibt einer Einschätzung zufolge das Problem bestehen, dass NGOs mit professionalisierten Strukturen nicht auf gleiche Weise für Betroffene sprechen oder diese aktivieren können, wie Mitgliederverbände. NGOs seien daher z. B. kein Ersatz für Gewerkschaften (Interview 30). Verstärkt wird dies dadurch, dass die zivilgesellschaftlichen Organisationen in der Tiefe als weniger stark ausdifferenziert angesehen werden als die Verbändelandschaft mit ihrer Vielzahl an Spezialisten-Verbänden (Interview 17). 


\section{Kooperation/Konfrontation}

Eng mit den Überlegungen zur zivilgesellschaftlichen Arbeitsteilung und zur ökologischen Nische in der Interessenlandschaft für das eigene Profil verbunden, ist die Unterscheidung von kooperativen und konfrontativen Handlungsformen. In der idealtypischen Unterscheidung dieser beiden Eckpunkte lassen sich verschiedene Ausprägungen finden, aus denen die Organisationen ihre strategische Ausrichtung auswählen. Ein erstes Kriterium beinhaltet Grundhaltung, Stil und Organisationsprofil. Die Spannweite beginnt bei Organisationen mit politiknaher Grundhaltung und moderaten politischen Forderungen. Diese Organisationen werden u. a. in Gremien und Kommissionen eingeladen und ähneln in ihrem Auftreten den Akteuren der verbandlichen Interessenvertretung. Ihr moderater Stil wird als Teil der Professionalisierungstendenzen verstanden. Über diesen moderaten Stil können diese NGOs teilweise auch weitreichende Forderungen aufstellen, selbst wenn diese eher diplomatisch formuliert werden (Interview 19, 29, 3, 23, 15). Auf der anderen Seite des Spektrums finden sich NGOs, die als öffentlichkeitszentriert und weniger politiknah charakterisiert werden. Sie werden selten in politische Entscheidungs- und Beratungsgremien eingeladen bzw. nehmen nicht daran teil. Stattdessen konzentrieren sie sich auf stärker zugespitzte, lautere, kontroverse und kampagnenfähige Positionen (Interview 23, 19,18).

Mit Blick auf den breiteren Kontext der NGO-Landschaft überwiegt in den Interviewaussagen die Einsicht, dass beide idealtypischen Formen situationsabhängig benötigt werden. Dies zeige sich bspw. daran, dass Organisationen oftmals auf breite Bündnisse setzen, um ihre Forderungen zu verfolgen (Interview 19). Der Stil der Handlungsformen ist jedoch nicht statisch, sondern wird nach Themen und situationsbedingt abgewogen. Bildhaft ist die Gegenüberstellung von Florett und Säbel (Interview 29, 3, 17).

Ein weiteres Kriterium für Grundhaltung und Stilauswahl ist die Austauschund Dialogorientierung der NGOs. Laut Interviewaussagen herrscht hier die Sichtweise vor, dass Protest allein nicht ausreiche, sondern dass Verständnis für verschiedene Gesichtspunkte im Gespräch entwickelt werden müsse. Insbesondere Themenallianzen legen einen moderaten Stil nahe und mäßigen die Positionszuspitzung (Interview 31, 22, 3). Dies kann sich zudem in der öffentlichen Wahrnehmung der NGO-Wirkung zeigen. Auch wenn häufig der Zusammenhang von lautem Auftreten und öffentlicher Erfolgswahrnehmung als ausschlaggebend angesehen werde, wäre es ein Trugschluss anzunehmen, dass es nicht auch leisen Organisationen gelinge, über Fachkompetenz und politische Anschlussfähigkeit Wirkungen zu erzielen (Interview 31). 
Die Orientierung an einem moderaten Stil geht laut mehreren Interviewaussagen so weit, dass die Organisationen teils die Implementationsperspektive politischer Akteure übernehmen und - analog zu großen Verbänden - in den Kategorien der verwaltungspolitischen Umsetzbarkeit denken. Dies wiederum stellt für die Organisationen eine neue Herausforderung dar. Im Spagat zwischen der (eher moderaten) Inside-Strategie mit Gestaltungswirkung und der (eher zugespitzten) Outside-Strategie mit Mobilisierungswirkung müssen sich die Organisationen glaubhaft und nachvollziehbar im Dreieck von Basis, Politik und Medien positionieren. Andernfalls kann die Organisationsspitze einen politischen Kompromiss nicht erfolgreich gegenüber ihren Mitgliedern bzw. Unterstützer*innen vermitteln (Interview 5, 29, 14).

Finanzielle Erwägungen sind ein weiteres Auswahlkriterium für Organisationsstil und -profil. Auf der einen Seite ermöglicht ein moderater Stil, von Finanzierungspartner*innen positiv wahrgenommen zu werden. Radikalere Handlungsstile finden sich demgegenüber eher bei Organisationen, die unabhängig von zusätzlichen Sponsor*innen sind. Insbesondere etablierte Mitgliederorganisationen sind aufgrund der Mitgliedschaftsbeiträge nicht auf externe Mittel angewiesen (Interview 9, 27). Auf der anderen Seite steht ein konfrontativer Stil, der mit politischer Sichtbarkeit und Mobilisierungswirkung für übergeordnete, als nicht-verhandelbar angesehene Ziele einhergeht. Wenngleich sich hieraus Mobilisierungspotenziale ergeben, kann ein Beharren auf radikalen Positionen auch Mitglieder, Spender*innen und Förderpartner kosten, wenn die politische Wirkung über lange Zeiträume ausbleibt. Deswegen gibt es eine grundlegende Tendenz zur Kompromissfähigkeit, um über den Weg der politischen Anbindung eine gesteigerte Chance auf Wirkung zu erzielen (Interview 31, 29).

\section{(Politische) Wirkung und kritische Sicht der eigenen Grenzen}

Die Interviews dokumentieren eine differenzierte und reflektierte Wahrnehmung der politischen Wirkung der NGO-Arbeit und eine kritische Sicht der eigenen Grenzen. Das Hauptargument hierfür ist, dass trotz der langjährigen Tätigkeit der NGOs, trotz kleinerer Fortschritte, trotz hohen Medienechos und relativ hoher gesellschaftlicher Akzeptanz für viele Forderungen, die zentralen Kernforderungen nicht durchgesetzt werden konnten.

Besonders deutlich zeigt sich dies daran, dass es - zum Zeitpunkt der Interviewdurchführung und Projektbearbeitung - keinen Politikwandel bei den Kernthemen der ausgewählten Organisationen gab. Im Fall von Attac gibt es keine eingeführte Finanztransaktionssteuer. Foodwatch konnte die geforderte, gesetzlich-verpflichtende Lebensmittelampel ebenfalls nicht durchsetzen, auch wenn die Einschätzung besteht, dass sich die Debatte über Lebensmittel und die 
politische Rolle von Essen verändert hat (Foodwatch 2019b, Die Gesellschafter 2009a). Zudem wurde im Herbst 2020 eine Verordnung beschlossen, durch die die freiwillige Anwendung des Kennzeichnungssystems „Nutri-Score“ eingeführt wurde. Im Fall von LobbyControl gab es zuletzt Bewegung im politischen Prozess. Nach einer Reihe von Skandalen im Feld der politischen Korruption und der illegitimen Einflussnahme löste sich die langjährige Blockadehaltung bei CDU/CSU. Im März 2021 einigten sich die Koalitionsfraktionen auf ein verpflichtendes Lobbyregister, welches im internationalen Vergleich im mittleren Regulierungsniveau zu verorten ist (vgl. Schiffers 2018b). Es soll zu Beginn des Jahres 2022 starten. Diese Entwicklung kam in ihrer Dynamik unerwartet, obwohl es kleinere Erfolge und eine zwischenzeitlich breite Koalition aus NGOs, Wirtschaftsverbänden und dem Berufsverband der Politikberatung ,degepol“ gab (Interview 30; zum Thema Lobbyregister, siehe Schiffers 2018b). In der Zusammenschau weist die Policy-Forschung jedoch nach (vgl. Baumgartner et al. 2009, Rüb 2014, Cairney 2019), dass diese inkrementalen Zwischen- bzw. Teilerfolge nicht darüber hinwegtäuschen dürfen, dass substanzieller Politikwandel zumeist ausbleibt.

Es wird insofern deutlich, dass bei der Bewertung des politischen Erfolgs unterschiedliche und teils voneinander abweichende Erfolgskriterien angelegt werden. Als ein Maßstab gilt das Ausmaß des erreichten Politikwandels im Sinne von inhaltlichen Policy-Zielen. Ein anderer sind Erfolg und Sichtbarkeit der Kampagnenarbeit (Speth/Zimmer 2015, Interview 5, 11). Da der Politikwandel in den Kernthemen oftmals ausblieb, sehen die Interviewpartner*innen die größten Erfolge vor allem im Bewusstseinswandel in der öffentlichen Debatte, im Kreis der Basis und teilweise der politischen Akteure (Foodwatch 2019b, Interview 6, 30, 5, 20, Die Gesellschafter 2009a). Diese Unterstützungsmobilisierung zieht allerdings nicht automatisch auch konkrete politische Erfolge nach sich (Interview 5). Die ausbleibende politische Wirkung führt in der NGO-Szene teils zu Enttäuschung (Interview 31, vgl. Frantz/Martens 2006: 65, 68-69). Einer Einschätzung zufolge liegt dies auch an Verzögerungstaktiken der Gegenseite, die den angestrebten Politikwandel verhindern möchten. Nebelkerzen und andere rhetorische Mittel können für Unklarheiten im Informationsprozess gegenüber der Öffentlichkeit führen, exemplarisch durch den Vorwurf, dass bestimmte Forderungen und Maßnahmen der NGOs zu unkonkret seien oder die politischen Ansatzpunkte und Verantwortlichkeiten unklar wären (Interview 2). Dennoch wird die Strategie, politischen Druck auf Entscheidungsträger*innen aufzubauen, als eine zentrale Bedingung für Wandelprozesse angesehen (Interview 8, 26, 9). Allerdings wird 
auch das Argument vertreten, dass es in bestimmten Situationen leichter fällt, politische Entscheidungsträger*innen zu überzeugen, wenn insbesondere auch leise, expertisebasierte Strategien verfolgt werden (Interview 31, 3, 11)

Themenkomplexität erweist sich als eine zentrale Herausforderung dabei, die Öffentlichkeit oder die eigenen Unterstützer*innen von der Bedeutung eines politischen Themas zu überzeugen (Interview 6, 8). Insofern zeigen sich hier klassische Mobilisierungsprobleme, die sich ebenso bei anderen Interessenvermittlungsorganisationen finden lassen. Deshalb erweist sich Erwartungsmanagement in der NGO-Arbeit zwischen den erreichbaren Zielen und einem unrealistischen Erwartungshorizont als notwendig. Auf diese Weise können die Unterstützer*innen ein Bewusstsein dafür entwickeln, auch kleine Erfolge in Richtung Politikwandel anzuerkennen, da andernfalls Enttäuschung droht (Speth/Zimmer 2015, Interview $31)$.

Zur Relevanz von Themenallianzen und Kooperationen mit großen Organisationen für größere politische Wirksamkeit herrscht eine geteilte Einschätzung. Zwar sind diese Formate gut geeignet, um Sichtbarkeit und punktuelle Aufmerksamkeit zu erzeugen. Allerdings täuscht das Format teilweise darüber weg, dass die meisten NGOs im Themenfeld relativ schwach ausgestattet und klein sind (Interview 30; siehe Kapitel 2 zur Diskussion der ,weapons of the weak"). Dies entkräfte, einer Einschätzung zufolge, das vereinfachende Bild des Machtgleichgewichts zwischen starken wirtschaftlichen Interessen und dem vermeintlichen großen Gegengewicht der NGOs. Gewicht auf die Waage bringen vielmehr einzelne große, international agierende Umwelt-NGOs mit besserer Personalausstattung.

Grenzen der eigenen Wirksamkeit zeigen sich u. a. bei der Inszenierung von Erfolgen. Kritisch gesehen wird, dass Organisationen versuchen, sich Erfolge auf die eigene Fahne zu schreiben, die auf gemeinschaftlichen Mobilisierungsanstrengungen beruhen oder ungeplant-zufälligen Themenkonjunkturen folgen (Interview 20, 14). Die Konkurrenz um individuell zuschreibbare Erfolge schließt an die Abwägung zur zivilgesellschaftlichen Arbeitsteilung und zur interessenlandschaftlichen Nische an.

Zur kritischen Sicht gehören auch strategische Herausforderungen und organisationsinterne Begrenzungen. Schwierig sei der Spagat zwischen Expertise- und Kampagnenarbeit einerseits und Mitgliederkommunikation andererseits. Obwohl der dezentralen Arbeit jenseits der öffentlichen und medialen Wirkung eine große Bedeutung zugeschrieben wird, tun sich verschiedene NGOs schwer damit, entsprechende Strukturen umzusetzen (Interview 30). Einigen Einschätzungen zufolge stehen bei bestimmten NGO-Typen vielmehr organisationserhaltende Interessen, wie Fundraising und Unterstützungsgenerierung, im Vordergrund, 
während die Interessenvermittlung - um einen politischen Konsens herzustellen - hinter Mobilisierung und Themensetzung zurückfällt (Interviews 31, 23). Zusammengenommen wird die unterstellte große Macht von NGOs stark relativiert.

\subsection{Auswertung der materialbasierten Kategorie (2) Themenwahl}

Das zweite der vier materialbasierten Kategoriensets liegt ebenfalls im Überschneidungsbereich des Dreiecks der Handlungsrationalität. Das Set gliedert sich in allgemeine Kriterien der Themenwahl, in den Bereich Agenda Setting und Kampagnenarbeit, Basis-Input, Mitgliederkommunikation, mediale Konjunkturen und Medienlogik sowie in finanzielle Abwägungen (Abbildung 5.9).

\begin{tabular}{|ll|}
\hline$\equiv=$ & $\begin{array}{l}\text { Kategorienset der Themenwahl } \\
\text { mit Kategorien Kriterien der Themenwahl, Agenda Setting und } \\
\text { KZ二 } \\
\text { Kampagnenarbeit, Basis-Input, Mitgliederkommunikation, mediale } \\
\text { Konjunkturen und Medienlogik sowie finanzielle Abwägungen }\end{array}$ \\
\hline
\end{tabular}

(Grafik: eigene Darstellung)

Abbildung 5.9 Kategorienset der Themenwahl

Bei Attac steht die Themenwahl im Zeichen des Basis-Inputs (siehe Infobox in Abschnitt 5.1). Themenvorschläge werden von Aktiven in Regionalgruppen oder inhaltlichen Arbeitsgruppen vorbereitet. Diese werden - zusammen mit weiteren Impulsen - für die Schwerpunktauswahl beim jährlichen Treffen des Entscheidungsgremiums „Ratschlag“ eingebracht und basisdemokratisch abgestimmt. Auf diese Weise sind die Schwellen für Engagement und thematische Rückkopplung sehr niedrig. Dennoch dreht sich der gewachsene Themenpool sehr stark um Finanzmärke und alternative Gestaltungsmöglichkeiten. Dort liegen in der eigenen Einschätzung und in der medialen Zuschreibung die wahrgenommenen Kompetenzschwerpunkte.

LobbyControl orientiert sich bei der Themenwahl in erster Linie an der eigenen Fachexpertise sowie den Kernanliegen zu größerer Lobbying-Transparenz und der Stärkung unterrepräsentierter Interessen (siehe Abschnitt 5.4). Für die Kampagnenauswahl müssen die (begrenzten) Kapazitäten der Geschäftsstelle und 
die thematische Expertise aufeinander abgestimmt sein. Gerade bei neuen, unbekannteren Themen dienen Kurz-Studien oder Diskussionspapiere als Einstieg in das Agenda-Setting. Die wahrgenommene Expertenstellung in der Öffentlichkeit trägt im Falle von medialer Aufmerksamkeit dazu bei, dass die Organisation direkt von Journalist*innen angefragt wird, wenn es etwa um den Seitenwechsel von Politiker*innen in die Wirtschaft oder Interessenvertretung geht. Die thematische Rückkopplung an die Basis erfolgt größtenteils indirekt über Zustimmung und Unterstützung durch Kommentare, Spenden und Fördermitgliedschaften. Gleichwohl werden weitergehende Überlegungen formuliert, wie diese Rückkopplungsschleife institutionalisiert werden könnte.

Für Foodwatch steht die „Kampagnenarbeit zur Stärkung der Verbraucherrechte" (Foodwatch 2019b) im Zentrum der Themenwahl. Sie dient dazu, das Bewusstsein für Transparenz im Lebensmittelmarkt, für Gesundheitskosten durch falsche Ernährung bei Verbraucher*innen zu schaffen und zur Mobilisierung beizutragen (Die Gesellschafter 2009a, Foodwatch 2019b). Um die politischen Forderungen nach verbraucher*innenfreundlicheren Regulierungen in die Öffentlichkeit zu bringen, nutzt die Organisation verstärkt den Weg der Konfrontation. Expertise in Form von Studien und Reports (siehe Abschnitt 5.6) liefert als Mittel der Öffentlichkeits- und Pressearbeit einen Kommunikationsanlass. Daher wird die inhaltliche Hauptarbeit der Teams Recherche, Kampagnen und Öffentlichkeitsarbeit eng abgestimmt. Neben dieser grob strukturierten Themenplanung zu den Kernanliegen setzt Foodwatch sehr stark auf spontane Aktion und Reaktion, um auch kurzfristig auf Lebensmittelskandale und die politische Konjunktur reagieren zu können. Aus dieser Orientierung resultiert auch die flexible Organisationsstruktur mit agiler, autonomer Spitze. Die Themenrückkopplung erfolgt indirekt und punktuell über Kommentare, Hinweise und vereinzelt Themenvorschläge der Unterstützer*innen.

Campact orientiert sich bei der Themenwahl an der Konjunktur politischer Entscheidungen, die auf der Kippe stehen (siehe Abschnitt 5.4 und siehe Infobox in Abschnitt 5.1). Da sich die Organisation explizit als Ergänzung zu FachNGOs versteht, hat sie keinen festen Themenpool. Im Allgemeinen entstammen die bearbeiteten Themen dem progressiven Milieu, um über die Beteiligungsund Protestmobilisierung die strukturellen Nachteile bei Themen der ,schwachen Interessen“, wie Menschenrechte, Umwelt- und Verbraucherschutz, abzumildern. Für die Themenfindung setzt die Kampagnenorganisation auf ein mehrstufiges Verfahren mit verschiedenen Auswahlschritten. Durch die Breite an Kampagnenthemen können sich die Unterstützer*innen insbesondere auch punktuell und kurzfristig engagieren, ohne langfristige Bindungen eingehen zu müssen. 
Für die Deutsche Umweltstiftung richtet sich die Themenwahl nach der eigenen Satzung und dem Ziel der Bildungs- und Informationsarbeit, um gesellschaftliche Veränderungen durch individuelle Verhaltensänderungen erzielen. Darüber hinaus lassen sich aufgrund des begrenzten Datenmaterials keine weiteren Kriterien ausmachen.

\section{Kriterien der Themenwahl}

Für die allgemeine Themenwahl zeigt sich in den Interviewaussagen die grundlegende Notwendigkeit für Relevanzkriterien, da es mehr potenziell relevante Themen gibt, als von einer Organisation bearbeitet werden können. Für die Themenauswahl stehen konkurrierende Kriterien zur Verfügung: Ein zentraler Faktor ist die (1) Auswahl nach Organisationsziel und Mission. Die Frage ist hier, ob ein Thema zur Organisation passt, z. B. über eigene Fachexpertise, über den Markenkern oder über die Mobilisierbarkeit der Unterstützer*innen. Entscheidend sind zudem die eigenen Kompetenzen, die Expertise zu den eigenen Schwerpunktthemen sowie die wahrgenommene Expertenstellung in der Öffentlichkeit und der Interessenlandschaft (Interview 6, 19, 30). Jahrelanges, themenbezogenes Arbeiten fördert die eigene Kompetenz im Sinne einer Rolle des politischen Spezialisten. Diese Rolle wird analog zur Themenkompetenz der Fachleute in Parteien und Verbänden gesehen (Interview 30). Die Mitarbeiter*innen der Geschäftsstelle benötigen thematisch-inhaltliche Motivation (Interview 16) (Abbildung 5.10).

Entscheidend wird die Themenwahl von Kriterium (2) der Wertegrundlagen und dem Selbstverständnis der NGO bestimmt. Grundsätzlich müssen Themen - aber auch Kooperations- und Finanzierungspartner - zu Profil, Werten und Anliegen der Organisation passen (Interview 21, 31, 8, 26). Nach außen drohen bei fehlender Passung Glaubwürdigkeits und Reputationsverlust. Nach innen müssen die Themen und Strategien mit den eigenen Überzeugungen von Team und Basis übereinstimmen. Wie bei anderen Organisationen auch spielt die Geschäftsführungspersönlichkeit eine Rolle, auf welche Weise die NGO in Erscheinung tritt.

Ein drittes Kriterium bezieht sich auf die (3) prozessuale Abwägung im Politikgestaltungsprozess. Geklärt werden muss die Themenrelevanz für die politische Öffentlichkeit und politische Entscheidungsträger*innen, wie das Thema komplexitätsreduziert als Konflikt inszeniert werden kann. Weitere Kriterien umfassen (4) die Abwägung, wie viel inhaltlichen Einfluss Mitglieder gegenüber der Organisationsspitze haben (siehe Basis-Input und Mitgliederkommunikation), die (5) ressourcenbezogene Abwägung, wie die finanziellen und personellen Kapazitäten wirkungsvoll eingesetzt werden können, (6) die Abwägung entlang 


\begin{tabular}{|c|c|}
\hline \multicolumn{2}{|l|}{$\begin{array}{l}\text { Auswahl- und Relevanzkriterien bei der Themenwahl } \\
\text { (hypothesengenerierende Typenbildung) }\end{array}$} \\
\hline \multirow{2}{*}{$\begin{array}{l}\text { 1. Auswahl nach Organisationsziel und Mission } \\
\text { 2. Auswahl nach Wertegrundlagen und Selbstverständnis } \\
\text { 3. Prozessuale Abwägung im Politikgestaltungsprozess } \\
\text { 4. Abwägung nach Mitglieder-/Basisbeteiligung } \\
\text { 5. Abwägung nach Organisationsressourcen und }\end{array}$} & $\begin{array}{l}\text { Ausdifferenzierung für } \\
\text { Agenda Setting und } \\
\text { Kampagnenarbeit }\end{array}$ \\
\hline & $\begin{array}{l}\text { Ausdifferenzierung für } \\
\text { Basis-Input }\end{array}$ \\
\hline $\begin{array}{l}\text { 5. Abwägung nach Organisationsressourcen und } \\
\text {-kapazitäten }\end{array}$ & $\begin{array}{l}\text { Ausdifferenzierung für } \\
\text { Mitgliederkommunikation }\end{array}$ \\
\hline $\begin{array}{l}\text { 6. Abwägung nach Medienlogik } \\
\text { 7. Abwägung nach Fundraising-Kriterien } \\
\text { 8. Abwägung nach Wirkungskriterien }\end{array}$ & $\begin{array}{l}\text { Ausdifferenzierung für } \\
\text { mediale Konjunkturen } \\
\text { und Medienlogik }\end{array}$ \\
\hline 9. Abwägung nach handhabbarer Anzahl von Themen & $\begin{array}{l}\text { Ausdifferenzierung für } \\
\text { finanzielle Abwägungen }\end{array}$ \\
\hline
\end{tabular}

(Grafik: eigene Darstellung)

Abbildung 5.10 Abstrahierte Kriterien zur Themenwahl von NGOs in der politischen Interessenvermittlung

der spezifischen Medienlogik (siehe Agenda Setting und Kampagne), die (7) Fundraising-bezogene Abwägungen und (8) wirkungsbezogene Abwägungen.

Mehrfach wird die Einschätzung vertreten, dass die (9) Glaubwürdigkeit und die Markenidentität einer Organisation darunter leiden kann, wenn zu viele Themen gleichzeitig bearbeitet werden. Hier besteht die Gefahr, dass das Organisationsprofil verschwimmt und die Organisation als beliebig wahrgenommen wird. Dies verschrecke Mitglieder. Im besten Falle entsteht eine Balance bei Organisationen, deren Ressourcenausstattung kontinuierlich wächst und die nach und nach mehr Themen bearbeiten können. Das Bewahren des Markenkerns gilt als großes Asset (Interview 10, 30).

\section{Agenda Setting und Kampagne}

Ein zentrales Kriterium für die Themenwahl sind die Anforderungen des agenda setting und der Kampagnenarbeit. In den Aussagen der Interviewpartner*innen findet sich eine Differenzierung zwischen Öffentlichkeitsarbeit und Medienstrategie auf der einen Seite sowie Campaigning auf der anderen Seite. In den Bereich der professionalisierten Öffentlichkeitsarbeit und Medienstrategie fällt die 
alltägliche und begleitende Kommunikation, die im innerorganisationalen Entscheidungsprozess verankert ist (Interview 19, 10, Die Gesellschafter 2009a). Im Mittelpunkt steht die langjährige Facherfahrung der Mitarbeiter*innen. Zu den Kommunikationsinstrumenten zählen selektive Pressekonferenzen, Medienarbeit mit Statements und O-Tönen. Auf diese Weise werden die politischen Forderungen inhaltlich zugespitzt, komplexe Themen in allgemeinverständliche Botschaften übersetzt und die Grundlage für die mediale Reputation der Organisation gelegt (Interview 21, 6, 15, 26, 5, 2, 14, 1, 13, 8, Die Gesellschafter 2009a). Besondere Bedeutung haben zudem Journalist*innenkontakte und Hintergrundgespräche, über die begleitende Fachexpertise vermittelt wird (Interview 29, 21, 5, 14, 3, 26, Speth/Zimmer 2015).

In den Bereich des Campaigning fällt die strategische Öffentlichkeitsarbeit mit dem Ziel, einen politischen bzw. gesellschaftlichen Wandel (Interview 19, 10) auf Basis der eigenen Expertise und der begrenzten Kapazitäten (Interview 26) zu erreichen. Nach den Intervieweinschätzungen lässt sich dieser Bereich in aktive und reaktive Elemente unterteilen. Als aktives Element funktioniert Campaigning über Fachexpertise als Kommunikationsanlass. Studien dienen dabei als Grundgerüst von Kampagnen. Daraus werden die Forderungen abgeleitet und auf hohem inhaltlichen Niveau unterfüttert (Interview 19, 26, 30, 13, 8, Foodwatch 2019b). Ein weiteres aktives Element läuft über die gezielte Thematisierung bisher vernachlässigter Themen. Kampagnen identifizieren bisher wenig beachtete, strukturelle Probleme und schaffen öffentliches Problembewusstsein (Interview 26, 30, 20, 8, Die Gesellschafter 2009a). Dies können beispielsweise Kosten gesellschaftlicher Probleme oder die Auswirkungen struktureller Zusammenhänge sein (Interview 20, Die Gesellschafter 2009a). Mittel hierzu sind Kooperation, Bündnisse und gemeinsame Kampagnen, um die Sichtbarkeit zu erhöhen (Interview 22, Die Gesellschafter 2009b), oder Plattformen, die eine Multiplikationsleistung erbringen (Interview 4).

Bei den reaktiven Elementen des Campaigning werden z. B. politische Entwicklungen herangezogen, wie Gesetzvorschläge, Ausschusssitzungen, oder politische Events genutzt, wie die Unterzeichnung von Koalitionsverträgen oder Klimakonferenzen (Die Gesellschafter 2009b, Interview 11, 3, 13, 1, 6, 30). Gleiches gilt für die Reaktion auf Skandale oder auf mediale Konjunkturen zum Organisationsthema, für die das Campaigning die inhaltliche Reputation der Organisation nutzt (Speth/Zimmer 2015, Die Gesellschafter 2009a, Interview 26, 30, $20,8,19)$. Als besonders hilfreich gilt, wenn das eigene Schwerpunktthema als Alleinstellungsmerkmal der Organisation gilt und sie dadurch ein starkes politisches Standing entwickeln kann (Interview 5). Die reaktiven Elemente können auch gezielt das Kommunikationsverhalten oder die Kommunikationsstrategien 
anderer Akteure aufgreifen (Interview 2, 30, 20). Beispielhaft festgemacht wird dies am Mobilisierungserfolg rund um den Hambacher Forst, der sich u. a. aus dem provokant wahrgenommenen Verhalten aus NRW-Landesregierung und RWE speiste (Interview 5, 14).

Es zeigen sich darüber hinaus die auch für alle anderen politischen Akteure limitierenden Faktoren eines aktiven Agenda-Settings. Zum einen ist der Raum für mediale und politische Aufmerksamkeit begrenzt (Die Gesellschafter 2009b). Daher schaffen es Themen häufig nicht auf die mediale Agenda (Interview 31). Zum anderen lassen sich komplexe Themen nur schwer kampagnenbasiert vermitteln (Die Gesellschafter 2009b). Hinzu kommen begrenzte personelle Kapazitäten für Expertisen und Medienanfragen (Interview 26, 30).

\section{Basis-Input}

Beim Basis-Input zur Themenauswahl lässt sich eine Bandbreite an grundlegenden Strategien unterscheiden. Sie reichen von idealtypischen bottom-up Prozessen bis hin zur nachträglichen Rückkopplung an die Unterstützungsbasis. Ein erster Typ ist ein stark basisorientiertes Vorgehen über Arbeitszusammenhänge der Ehrenamtlichen und Aktiven der Basis (siehe Infobox zu Attac, Abschnitt 5.1). Die Organisationsspitze schafft die Möglichkeit und Infrastruktur für Engagement mit möglichst niedrigen Beteiligungsschwellen, während die inhaltliche Richtung von der Mitgliederbasis festgelegt wird. Inhaltliche Vorschläge kommen teils direkt aus den eigenen Untergruppen und werden von der Organisationsspitze unterstützend flankiert (Interview 20, 4, 11, 6, 31).

Der zweite Typ ist ein moderat basisorientiertes Vorgehen über Kernthemen und Überzeugungen. Die Themenvorschläge kommen entweder direkt aus dem Kreis der (ehrenamtlich) Aktiven (Interview 20, 31) oder über Beteiligungsplattformen (siehe Infobox zu Campact, Abschnitt 5.1). Deren technologische Infrastruktur erlaubt es, Input zu erhalten, Präferenzen abzufragen und Testmöglichkeiten für das Ausmaß gesellschaftlicher Unterstützung für ein Thema zu schaffen (Interview 19). Der Typ umfasst auch die Möglichkeit, punktuelle Rückmeldung durch Zuschriften der Unterstützer*innen bzw. Mitglieder aufzunehmen (Interview 8, 9). Gerade die Finanzierung über Kleinspenden und Mitgliedsbeiträge wird als Teil der Legitimation gesehen (Interview 5, 31).

Ein dritter Typ ist das out-putorientierte Vorgehen. Die Organisationsspitze handelt im Sinne der satzungsmäßigen Organisationsziele und gibt transparente Betätigungs- und Leistungsnachweise über Erfolge, Aktionen etc. für die Mitglieder bzw. Unterstützer*innen. Die Rückmeldungsmöglichkeit ist ebenfalls punktuell möglich mit Themenwünschen durch Zuschriften der Unterstützer*innen (Interview 26, 21, 30, 23, 8, 25, Die Gesellschafter 2009a, 2009b). 
Eng damit verbunden ist der vierte Typ des nachträglichen Rückkopplungsmechanismus. Zustimmung, Unterstützung und Kritik über die geleistete Arbeit werden an Kleinspenden, Graswurzelfinanzierung, Crowdfunding, Spendenveränderungen sowie an Neumitgliedschaften und Austritten festgemacht (Interview 26, 21, 30). Dieser indirekte Mechanismus muss durch ausführliche Kommunikation begleitet werden, um Transparenz und Glaubwürdigkeit sicherzustellen. Das gilt insbesondere bei zentralen Konfliktthemen, bei denen die Kommunikation auch lautstark erfolgen muss, um die Erwartungen der Basis zu erfüllen (Abbildung 5.11).

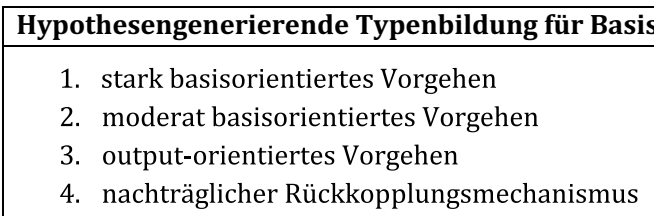

\section{Hypothesengenerierende Typenbildung für Mitgliederkommunikation}

1. über professionalisierte Kommunikationsstrukturen.

2. über formale Organisationsstrukturen

3. über persönlichen Austausch zwischen Organisation und Unterstützer*innen

4. über thematische Rückkopplung

(Grafik: eigene Darstellung)

Abbildung 5.11 Hypothesengenerierende Typenbildung für Basisinput und Mitgliederkommunikation

\section{Mitgliederkommunikation}

Für die Themenwahl werden zudem Kriterien für die wechselseitige Kommunikation mit Mitgliedern bzw. Unterstützer*innen herangezogen. Anhand der Interviewaussagen lassen sich vier Typen identifizieren. Der erste Typ ist die Mitgliederkommunikation über professionalisierte Kommunikationsstrukturen. Verwendet werden Kommunikationskanäle mit großer Reichweite. Diese umfassen klassische Wege, wie Jahresberichte, Webseiten, Newsletter und Social Media sowie neuere Wege, in denen das Nutzerverhalten aktiv ausgewertet und in die Kommunikation zurückgespielt wird (Interview 5, 14, 6, 26, 23, 21, 9). Die Grenze dieses Typs bilden zum einen personelle Kapazitäten und zum 
anderen die teils hohe Komplexität der Themen, die sich nicht für alle Kommunikationsformate eignet. Daher ist eine Abwägung zwischen Kampagnen-, Expertise- und Mitgliederkommunikationsarbeit nötig (Interview 5, 6, 30, 17). Die professionalisierten Kommunikationskanäle zeichnen sich z. B. durch die zielgruppenspezifische Gestaltung von Mitgliedermagazinen aus (Interview 22), sie geben Hinweis auf Möglichkeiten, um selbst aktiv werden zu können (Interview 20) und präsentieren die eigenen Kampagnenerfolge und -rückschläge. Vermehrt setzen die Organisationen darauf, die Wirkung des persönlichen Engagements aufzuzeigen (Interview 8). Mit diesem professionalisierten Kommunikationstyp sind Überlegungen für eigene Mitgliederentwicklungsstrategien und Basisstrategien verbunden, die den Kontakt mit der Basis systematisch stärken sollen (Interview 30). Die Hürden hierbei sind neue Hierarchien zwischen Organisationsspitze und Basis, die durch die Professionalisierung entstehen und den Austausch bspw. in Konfliktsituationen behindern können (Interview 12, 15).

Der zweite Typ der Mitgliederkommunikation läuft über formale Organisationsstrukturen. Im Zentrum stehen Delegiertenversammlungen und Kreis/Ortsgruppen bei Organisationen mit einer (public interest) Verbandsstruktur (Interview 5, 11, 19, 21). Diese Strukturen entsprechen der idealtypischen Vorstellung von Verbänden. Bei Organisationen ohne klassische Mitgliedschaft kann die Kommunikation allerdings ein Manko sein (Interview 28), da diese formalen Strukturen nicht vorhanden sind. Im Sinne von, form follows function " unterscheiden sich die Kommunikationskanäle bei Organisationen mit hohen und geringen Mitgliederzahlen (Interview 14). Entsprechend finden Mitgliederversammlungen bei NGOs oftmals eher in kleinem Rahmen statt (Interview 30).

Der dritte Typ der Mitgliederkommunikation setzt auf persönlichen Austausch zwischen Organisationspersonal und Unterstützer*innen. Die verwendeten Formate reichen von formalen Regionalkonferenzen bis hin zu informellen Stammtischen. Zudem dient die Kommunikation am Rande von Aktionen als Plattform für Austausch und Vernetzung (Interview 20, 15, 30). Das Ziel dieses Typs ist es, den Basisaustausch zu intensivieren und die Unterstützer*innen direkt zu aktivieren (Interview 30).

Der vierte Typ der Mitgliederkommunikation erfolgt über eine thematische Rückkopplung, um weiterführenden Input für die Organisationsarbeit zu generieren. Wie bei der Kategorie Basis-Input wird zum einen das Engagement der Aktiven in den formalen Organisationsstrukturen genutzt (Interview 11), zum anderen wird auch die Kommunikation in Social Media und via E-Mails herangezogen. Inhaltliche Anregungen der Basis bündeln sich jeweils insbesondere nach Aktionen (Interview 5, 26, 30, 8, 31). Einer Einschätzung zufolge eignen sich 
Newsletter hier nur begrenzt, um inhaltliche Rückmeldungen zu erhalten (Interview 2). Zudem wird die strukturelle Unterscheidung genannt, dass Mitglieder in diesem Kommunikationstyp im Gegensatz zu losen Unterstützer*innen weniger intensiv bei Laune gehalten werden müssen (Interview 25), da sie sich der Organisation stärker verbunden fühlen. Die bereits genannte, indirekte Rückmeldung über Mitgliederwachstum und -austritte (Interview 30) fällt ebenfalls in diesen Typ.

\section{Mediale Konjunkturen und Medienlogik}

Mediale Konjunkturen und Überlegungen zur Medienlogik wirken ebenfalls auf die Themenauswahl von NGOs. Als Erfolgsfaktoren für mediale Resonanz gelten ein niedriges Komplexitätsniveau, persönliche und emotionale Betroffenheit (im Alltag), hohe Sichtbarkeit (bei Demonstrationen), wahrgenommene Konflikte oder Krisensituationen, aufgedeckte Skandale und eigene Empörung bei den Journalist*innen. Dadurch werde es leichter, die Öffentlichkeit auf NGO-Themen anzusprechen, Handlungsdruck aufzubauen und Handlungsfenster zu nutzen. Problematisch seien Themen mit hohem Komplexitätsniveau, geringem öffentlichen Interesse und schwacher medialer Resonanz (Interview 26, 6, 30, 19, 29, 10, 12, Die Gesellschafter 2009b, Foodwatch 2009b). Mobilisierung und Medienresonanz können sich dadurch wechselseitig verstärken. Als Beispiele werden die Demonstrationen zu TTIP, am Hambacher Forst und die Fridays for Future genannt (Interview 22, 5, 20).

Allerdings werden auch die Grenzen aufgezeigt: NGOs seien bei der Aufdeckung von Skandalen oftmals von anderen Medien abhängig und könnten nicht schnell genug auf Empörungswellen reagieren (Interview 6, 20, 30). Daher gelten mediale Konjunkturen als begleitender Faktor und werden bei Überlegungen zu Themenwahl und Fundraising im Hinterkopf behalten (Interview 26, 12).

Der Medienlogik folgend wird die Themenwahl von Überlegungen zur bereits genannten Emotionalisierung sowie zur Gewinnbarkeit, Vereinfachung und „Skandalisierung“ getragen (vgl. Bautz 2008: 108-110). Das Auswahlkriterium der Gewinnbarkeit richtet sich an Themen, bei denen auf bestimmte Art und Weise Erfolge erzielt werden können. Auch hier reflektieren die Interviewpartner*innen die eigenen Grenzen kritisch: Nicht alle Themen sind medial gewinnbar, oftmals brauche es eher Verhandlungen und persönliche Kontakte (Interview 19, 31, 26, 29). Für die Gewinnbarkeit müssen politische Chancen für Veränderung vorhanden sein, die in breiten Kreisen als politisch relevant und drängend angesehen werden. So können vorhandene Stimmungen in Bevölkerung und Medien für ein Anliegen kanalisiert werden (Interview 19, 17, Die Gesellschafter 2009b). In bestimmten Situationen können Organisationen Erfolge präsentieren, wenn sie 
sich auf klar begrenzte Themen mit Symbolwirkung als „low hanging fruits“ konzentrieren (Interview 17).

Für den weiterführenden Kontext formulieren die Interviewpartner*innen das Auswahl- und Abwägungskriterium der Vereinfachung. Wie bereits genannt, tragen verständliche Botschaften zu klar heruntergebrochenen Konfliktthemen auf niedrigem Komplexitätsniveau dazu bei, die Unterstützer*innen und die Öffentlichkeit zu aktivieren. Dabei helfe die holzschnittartige Unterscheidung zwischen „Gut und Böse“ bzw. „David gegen Goliath“ (vgl. (vgl. Berg 2016, Bayerischer Rundfunk 2017), um das Thema in eine bildhafte, personalisierte Geschichte einzubetten (Interview 2, 4, 22, 1, 24, Die Gesellschafter 2009b). Hier muss die Abwägung getroffen werden, ob Themen, die eine Organisation für relevant hält, auch kampagnenfähig sind oder ob sie hinter besser geeignete Themen zurückfallen müssen (Interview 19, 30, Die Gesellschafter).

Ebenfalls allgemein formuliert und als differenziert betrachteter Begriff wird das Kriterium der „Skandalisierung“ genannt (vgl. Bautz 2008: 108-110). Zentral für das Kriterium ist das hohe Maß an öffentlicher Aufmerksamkeit. Unterschieden wird jedoch, ob ein Thema durch aufgedeckte politische Skandale oder Krisensituationen erscheint (z. B. Lebensmittelskandale, politische Korruption), ob ein aktuelles Thema durch Kampagnenarbeit und emotionale Mobilisierung hochgezogen wird oder ob kreative Aktionen und prägnante Botschaften zu medialer Sichtbarkeit verhelfen (Interview 19, 1, 24, 20, 30, 12). Kritisch gesehen wird, wenn Organisationen die medialen Wellen der Berichterstattung nutzen, um „Agenda-Surfing“ zu betreiben (Interview 23). Abseits der Kernthemen kann ein häufiger Themenwechsel als willkürlich und opportunistisch aufgefasst werden.

\section{Finanzielle Abwägungen}

Die Themenwahl wird auch von Finanzierungserwägungen beeinflusst. Hier zeigt sich eine reflektierte Haltung zu Abhängigkeiten und Zwängen, die sich in den Interviewaussagen findet. Ein hoher Anteil an Mitgliedsbeiträgen (Voll- und Fördermitgliedschaften) bei der Finanzierung gilt allen Organisationen als Sicherung der inhaltlichen und thematischen Unabhängigkeit und um ggf. Interessenkonflikte mit anderen Finanzierungspartner*innen zu vermeiden (Interview 26, 30, 20, 11, 12, 19, 27, 31 Foodwatch 2019b, Die Gesellschafter 2009a). Aus diesem Grund setzten NGOs auch auf Crowdfunding sowie projektgebundene oder ungebundene (Klein-)Spenden, die u. a. im Nachgang von Aktionen eingenommen werden können (Interview 19, 20, 26, 27, 9). Verschiedenen Einschätzungen zufolge haben Faktoren, wie Emotionalisierbarkeit, persönliche Betroffenheit, thematische Zuspitzungen und eine Konzentration auf ,schlechte Nachrichten ' einen hohen Fundraising-Erfolg (Interview 6, 27, 12, 24). 
LobbyControl habe laut Aussagen der Interviewpartner*innen bewusst eine Mitgliederentwicklungs- und Basisstrategie eingesetzt, um nicht einseitig von projektbezogener Finanzierung und von Großspenden abhängig zu sein. Foodwatch setzt ebenfalls auf eine Mitgliederbindungsstrategie für die Förder*innen (Foodwatch 2019b). Auch Attac ist sich bewusst, dass das eigene organisationale Überleben von den Beitragszahlungen der Mitglieder abhängt.

Hohe Einigkeit aller Interviewpartner*innen herrscht dahingehend, dass auch projektbezogene Finanzierungspartner*innen zum eigenen Profil und Selbstverständnis passen müssen (Interview 26, 6, 20, 21, 29). Dies gelte auch für kooperierende Stiftungen und Drittmittelpartner*innen (Interview 6,18). Gerade im Rahmen der Professionalisierung und in Phasen des Organisationswachstums müssen längerfristige Finanzierungsquellen aufgetan werden, u. a. um die steigenden Personalkosten decken zu können und Planungssicherheit zu schaffen (Interview 20, 8, 9).

Dennoch beeinflusse die Frage der Finanzierung zivilgesellschaftlicher Arbeit bewusst und unbewusst die Themensetzung und Projektumsetzung. Neben der eigenen Überzeugung, dass bestimmte Themen der NGOs politisch relevant sind, muss die Gesamtheit der behandelten Themen grundsätzlich dazu geeignet sein, Spenden und Neumitgliederinteresse zu generieren. Hier muss eine Abwägung zwischen der Einschätzung der politischen Relevanz des Themas und dessen Fundraising-Eignung getroffen werden (Interview 26, 12, 19). Gleichsam steht das Einwerben von Spenden einer Einschätzung zufolge unter einer erhöhten, kritischen Medienbeobachtung, da einzelne Spendenskandale das Vertrauen in den gesamten Spendenmarkt erschüttern (Interview 27).

\subsection{Auswertung der materialbasierten Kategorie (3) Expertise}

Das dritte der vier Kategoriensets thematisiert die Bereitstellung fachspezifischer Expertise durch NGOs. Diese Expertise dient allen drei Dimensionen des Dreiecks der Handlungsrationalität. Das Set gliedert sich in die Kategorien politisches Instrument, öffentliche Anerkennung, Erfolgsgrenzen der Fachexpertise-Strategie und Personalisierung von Expertise (Abbildung 5.12). Die allgemeine Einschätzung der Interviewpartner*innen ist, dass thematisch-fachliche Expertise als zentraler Profilschwerpunkt einer NGO eine hohe Bedeutung für den politischen Einfluss hat. Fachliche Expertise ist ein wichtiges Instrument, sowohl für die grundlegende Wahrnehmung als relevanter Gesprächspartner und politischer Akteur, als auch, um öffentliche Sichtbarkeit, Reputation oder Legitimation 
zu erreichen. Die inhaltliche Fachexpertise genieren die NGOs durch eigene Recherchen, Studien oder die Verarbeitung von Drittstudien.

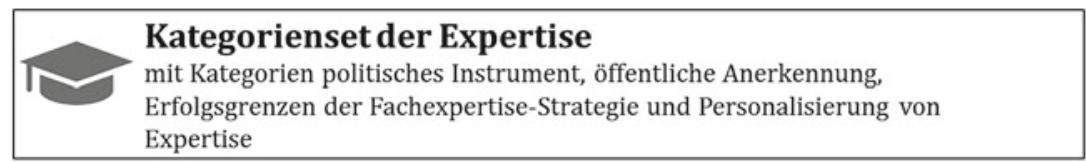

(Grafik: eigene Darstellung)

Abbildung 5.12 Kategorienset der Expertise

Neben der Fachexpertise wird allerdings betont, dass es auch andere relevante „Expertisen“ gibt, die eine Organisation aufbauen muss, um erfolgreich arbeiten zu können. Teilweise wird dieses Wissen als „Politikstrategie-Expertise“ (Interview 30) bezeichnet, als Kampagnenfähigkeit oder als professionelle Medienarbeit. Die untersuchten NGOs nutzen innerhalb ihrer strategischen Schwerpunktsetzung eine große Bandbreite von inhaltlichem Fachwissen bis hin zum Aufbau einer Protestplattforminfrastruktur ohne dezidiert-eigene thematische Expertise. Es herrscht die Einschätzung vor, dass jede Organisation eine Mischung von Expertise-Typen benötigt - ob im eigenen Haus oder speziell eingeworben (Interview 11, 30, Foodwatch 2019b). Denn Fachexpertise ohne Kampagnenexpertise wird als nicht zielführend angesehen - eine Kampagne ohne validen Inhalt ebenfalls nicht.

Laut Interviewaussagen nutzen LobbyControl, Attac und Foodwatch unterschiedliche Formen dieser Expertisestrategien, um ihre Ziele zu verfolgen. Während LobbyControl seine Fachexpertise häufig direkt als Angebot an Exekutive und Legislative adressiert (Parlamentsanhörungen, Gesetzentwürfe), legt Attac den Schwerpunkt auf Bildungsarbeit und auf das empowerment des Unterstützungskreises der „Attacies“, damit diese über informiertes Handeln Veränderungen im politischen Prozess erreichen können. Foodwatch nutzt die eigens generierte Expertise, um konfrontatives Agenda-Setting mit Handlungsempfehlungen zu verbinden und versteht die Veröffentlichung von Studien vor allem als Kommunikationsanlass und Startpunkt für eine darauffolgende Kampagne. Campact hingegen geht einen eigenen Weg und verzichtet auf die Etablierung eigener spezifischer Fachexpertise und holt diese, je nach Kampagne, über Kooperationspartner*innen ein. Die Organisation fokussiert sich auf die Funktion 
als Protestplattform, Verstärker und Kampagnenorganisation. Die OrganisationsExpertise liegt hier im Aufbau der Mobilisierungsinfrastruktur, Kampagnenlogistik und technischem Support. Die Deutsche Umweltstiftung greift augenscheinlich in ihren Projekten auch auf die Expertise von externen bzw. mit der Stiftung verbundenen Fachexperten zurück. Sichtbar ist dies am Beirat der nachhaltigkeitsorientierten Crowdfunding Plattform „Ecocrowd“ oder bei der Kampagne \#kaufnix.net.

\section{Politisches Instrument}

Fachstudien, spezifische Informationen und Expertisen sind etablierte Instrumente in der Interessenvertretung und werden vorwiegend als wertvolles politisches Gut im Tauschgeschäft des klassischen Inside Lobbyings verstanden (Berkhout 2013). Fachexpertise und spezifische Handlungsempfehlungen werden in die politische Arena eingespeist, mit der Erwartung, dass sie sich in Gesetzgebung bzw. politischen Richtungsentscheidungen wiederfinden (Interview 26).

Durch die Interviews und Recherchearbeiten wurde allerdings deutlich, dass der Fachexpertise ein durchaus breiteres Funktionsspektrum zugeordnet werden kann. Zunächst muss hier die strategische Veröffentlichung von Expertise als Kommunikationsanlass genannt werden (Interview 8, 19, 17, 23). Organisationen sind sich bewusst, dass ihre Studien und Reports zwar von interessiertem (Fach-)Publikum und eventuell den zuständigen Mitarbeiter*innen in Ministerien gelesen werden. Unterstützer*innen und Medien nehmen allerdings eher die Zusammenfassungen und Pressemitteilungen darüber wahr. Dadurch hat sich ein ritualisiertes Verfahren etabliert, in welchem die mediale Veröffentlichung einer Studie als Auftakt und inhaltliches Fundament der darauf aufbauenden Kampagne fungiert. Aus der Studie werden konkrete politische Forderungen für das übergeordnete Ziel abgeleitet und mit Nachdruck in die Debatte eingespeist (Interview 19, 11). Unterstützer*innen erhalten Zugang zu vereinfachten Zusammenfassungen (executive summary), in denen Problemlagen, Handlungsdruck und Lösungsoptionen dargestellt werden. Diesem expertisebasierten Kampagnenaufschlag folgen öffentlichkeitswirksame Aktionen und Veranstaltungen - die Fachexpertise tritt in diesem Schritt zugunsten der Kampagnenarbeit in den Hintergrund. Diese instrumentelle Verwendung von Expertise folgt der Einsicht, dass interessanter Inhalt ein gewisses $\mathrm{Ma}$ an Aktionismus benötigt, um in der politischen Öffentlichkeit wahrgenommen zu werden (Interview 30).

Neben der thematisch-inhaltlichen Kompetenz merkten die Interviewpartner*innen mehrfach an, dass für bestimmte NGOs ein grundlegendes Verständnis als Dienstleister notwendig ist, um politischen Einfluss nehmen zu können. NGOs liefern - vergleichbar zu Verbänden - den politischen Entscheidungsträger*innen 
zeitnah Daten und Einschätzungen zu Politikauswirkungen, um politische Forderungen zu untermauern (Interview 23). Offen reflektiert wird die gemeinsam geteilte Einschätzung, dass jede Studie, die ins politische System eingebracht oder von politischen Entscheidungsträger*innen aufgenommen wird, einem unumgänglichen Bias unterliegt. Trotz valider wissenschaftlicher Methodik ist eine Studie immer unter der interessengeleiteten Perspektive des Auftraggebers zu interpretieren (Interview 2, 16, 30). Kurz: Die einstimmige Meinung der Interviewten ist, dass jede Studie in ihrer Wahrnehmung gefärbt ist. Dadurch bestehe die Gefahr, dass der politische Wert von Studien langfristig marginalisiert wird. Die relative Bedeutung und der komparative Mehrwert gehen verloren, wenn zu viele interessengeleitete Studien von zu vielen Akteuren kursieren. Statt fakten- und wissensbasierte Klarheit zu liefern, führe dies zu neuer Unübersichtlichkeit. Nichtsdestotrotz brauchen politische Entscheidungsträger*innen häufig nicht nur explizit-inhaltliche Expertise, um ihre Positionen auch innerparteilich (oder innerhalb des Kabinetts) durchzusetzen (Interview 2, 29), sondern zudem als Kulisse vieler glaubhafter Unterstützer*innen, die die Dringlichkeit eines Problems untermauern.

Auch wenn sich die Funktionen des politischen Instruments „Expertise“ durchaus erweitern, bleibt die Urangst vieler Organisationen, inhaltlich fehlerhafte Studien zu veröffentlichen (Interview 15, 16, Foodwatch 2019b). Der Schaden, den die Glaubwürdigkeit der Organisation erleidet, wäre höchst gefährlich. Zusammen mit den begrenzten finanziellen und personellen Ressourcen werden Expertisen als politisches Instrument nur sehr selektiv für die zentralen Kernthemen der Organisation verwendet. Ihr Einsatz erfolgt lediglich nach gründlicher Recherche und vielfacher Überprüfung der sachlichen Ausrichtung und solange ein Alleinstellungsmerkmal in der zivilgesellschaftlichen Konkurrenz um Aufmerksamkeit vorliegt (Interview 26).

\section{Öffentliche Anerkennung}

Zentraler Baustein für den guten Ruf von Fach-Organisationen sind qualitativ hochwertige, wissenschaftliche Erkenntnisse, um die eigene Argumentation zu untermauern (De Bruycker 2019). Schafft es eine NGO die Reputation als glaubwürdige Expertin zu etablieren, kann sie öffentliche Anerkennung erfahren. Dies zeigt sich u. a. daran, dass sie proaktiv von Medien angesprochen werden - teilweise auch mit der Bitte um Einschätzungen im weiter gefassten Umfeld der thematischen Kernkompetenz (Interview 21, 26, 6, 5, 11, Foodwatch 2019b). Die Expertenrolle hilft beim Kontaktaufbau mit Journalist*innen. Für die Medien eine Quelle für gut gebündelte Hintergrundinformationen zu sein, ist ein Zeichen 
der fachlichen Anerkennung. Allerdings, so die Einschätzung verschiedener Interviewpartner*innen, trägt diese zeitintensive Rolle nicht automatisch dazu bei, die Ziele der Organisationen zu erreichen (Interview 21, 26).

Interessanter ist es für die Organisationen, wenn sie die Medienlogik im Spiel der personalisierten Stellungnahmen nutzen können. Häufig werden NGOVertreter*innen als Gegenposition zu politischen Entscheidungsträger*innen inszeniert. Zugespitzt werden hier NGO-Vertreter*innen als ,alternative Politiker“ bezeichnet (Interview 15, 26). Diese erhöhte Sichtbarkeit verschafft den NGOs einen Vorteil in der Konkurrenz um Aufmerksamkeit. Die Inszenierung über die Bande steigert die Themenrelevanz auf der medialen Agenda (Interview 30).

Einschränkend wird angemerkt, dass der Aufbau von Reputation als glaubwürdiger Expertiseanbieter langwierig ist und kontinuierlich erhalten werden muss. Organisationen müssen sehr umsichtig bei der Wahl neuer Themen und bei der Erfolgsdarstellung sein. Opportunistisches Themensurfen, aber auch das erfolglose Festhalten an Maximalpositionen können als Willkürlichkeit ausgelegt werden und die Glaubwürdigkeit beschädigen (Interview 30, 21, 23).

\section{Erfolgsgrenzen der Fachexpertise-Strategie}

Der (Selbst-)Einschätzung der Interviewpartner*innen zufolge, garantiert fachliche Expertise allein keinen Erfolg bezüglich des angestrebten Politikwandels (Interview 26, 30). Keine der untersuchten NGOs konnte bisher die Kernforderung des Organisationsziels in Gänze umsetzen. Dies zeigt sich auch in den selbstkritischen Einschätzungen zur politischen Wirksamkeit (siehe Abschnitt 5.4). Die Forderungen nach einer gesetzlich verankerten Lobbyregulierung (LobbyControl) wurde im Nachgang an den Untersuchungszeitraum in einer moderaten Version teilweise erreicht. Bisher ausgeblieben sind eine Finanztransaktionssteuer (Attac) oder eine verpflichtende Lebensmittelampel (Foodwatch). Ohne den politischen Willen der Entscheidungsträger*innen in den hürdenreichen und langfristigen Politikwandelprozessen (vgl. Baumgartner/ Berry et al. 2009, Schiffers 2021) bleibt die Durchschlagskraft der Organisationen begrenzt. Die Expertisestrategie aus Agenda Setting und Handlungsempfehlungen muss daher mit der angesprochenen Umsetzungs- und Politikstrategie gekoppelt werden.

Dennoch gab es laut überwiegender Einschätzung der Interviewten sichtbare Erfolge beim Agenda Setting der Kernthemen der Organisationen (Interview 8, 30, 26, 11, 9). Die Diskussion um Transparenz in Gesetzgebungsprozessen ist ebenso etabliert wie die zunehmende Wahrnehmung der politischen Bedeutung von Ernährung. Auch die Finanztransaktionssteuer hat eine gewisse mediale Konjunktur. 
Einigkeit herrscht aber darüber, dass Agenda Setting nicht das einzige Organisationsziel sein kann. Keine der Organisationen versteht sich als reine AufklärerNGO, die Fachexpertise zum Selbstzweck generiert. Im Gegenteil, trotz der Rückschläge wollen alle fünf Organisationen ihre Forderungen politisch umsetzen. Ungeachtet der Legitimation durch Expertise (siehe Abschnitt 5.7), bleibt einer Einschätzung zufolge das Problem der übergeordneten Strategie (siehe Abschnitt 5.4), dass NGOs - anders als Mitgliedschaftsverbände - direkt betroffene Menschen nicht direkt organisieren können. Als Organisationstyp sind NGOs daher kein Ersatz für Mitgliedschaftsverbände (Interview 30).

Begrenzt wird die Expertisestrategie ebenso dadurch, dass die mediale Aufmerksamkeit sehr selektiv und auf wenige Themen fokussiert ist. Daher ist es schwierig, selbst mit den Hauptthemen sichtbar zu bleiben. Windows of Opportunities - ausgelöst durch Skandale, Katastrophen oder andere unerwartete Entwicklungen - können Themen und Entscheidungen triggern, an denen Organisationen trotz jahrelanger Vorarbeiten scheiterten (Interview 11, 6).

Eine weitere Grenze der Expertisewirkung liegt laut Interviewaussagen in den eingeschränkten Ressourcen. Zwar können sich NGOs häufig langfristiger mit einem Thema beschäftigen, als es Journalisten*innen und Medien vermögen. Allerdings, so die Einschätzung, bleiben sie auf vertiefende und kontinuierliche Forschung aus dem Bereich der Wissenschaft angewiesen. Für die NGO-Arbeit stünden hierfür weder ausreichende Mittel zur Verfügung, noch entspricht es der Aufgabe und dem Selbstverständnis der Organisationen. NGOs sind eben keine wissenschaftliche Einrichtungen oder Think Tanks (Interview 26, 30, 5). Einer Einschätzung nach müsse auch zur Expertise selbst Transparenz herrschen, um überhaupt die Chance zu haben, Expertise hinterfragen zu können (Interview 30).

\section{Personalisierung von Expertise}

Der Trend zur Personalisierung von Expertise (Hilton/McKay et al. 2012) beschreibt, wie NGOs ihre Relevanz erhöhen, indem sie im Markt der ausdifferenzierten Expertise mit professionalisierten Medien- und Mitgliederstrategien agieren. Der übereinstimmenden Einschätzung der Interviewpartner*innen zufolge, entspreche eine solche Strategie der medialen Logik zur Vereinfachung komplexer Zusammenhänge. In der andauernden Konkurrenz um öffentliche Aufmerksamkeit könne dies einen erheblichen Vorteil ergeben, wenn eine vertraute Person als Gesicht der Organisation ad personam stilisiert werde und auf diese Weise Glaubwürdigkeit und Zuspruch generiert (Interview 30, 12, 18, 21).

Dennoch wird die übermäßige Personalisierung nicht als langfristig sinnvolle Strategie wahrgenommen. Trotz punktueller Effekte mit hoher medialer Sichtbarkeit einzelner Gesichter als Identifikationsfigur, wird die Personalisierung 
ambivalent bewertet (Interview 30, 26, 23). Grundsätzlich wird das Risiko, dass Reputation und Unterstützung nach dem Ausscheiden einer solchen Person verloren gehen können, als zu groß eingeschätzt (Interview 23, 6, 11). Auch wird die Gefahr gesehen, mithilfe hoher finanzieller Mittel Personen als vermeintliche Experte*in künstlich zu inszenieren und damit dem Einfluss von außen die Türen zu öffnen (Interview 30).

Laut Einschätzung der Interviewpartner*innen arbeiten viele NGOs daran, dass ihre Organisation unabhängig vom Spitzenpersonal ein hohes Maß an Glaubwürdigkeit und inhaltlicher Reputation erlangen. Ein Weg hierfür ist, dass möglichst viele Mitarbeiter*innen medial ,sprechfähig“ sind. Damit können - abhängig vom jeweiligen Thema - die zuständigen Fachreferent*innen zu Wort kommen, anstatt die Medienarbeit komplett dem Kopf der Geschäftsführung zu überlassen (Interview 8, 6, 20). Der Aufbau medial sprechfähiger Experten*innen ist, unabhängig von der Anzahl, allerdings nur von hauptamtlichen Mitarbeiter*innen zu leisten.

Trotz dieser durchweg reflektierten Wahrnehmung der Chancen und Risiken der Personalisierung von Expertise wird die Rolle von persönlichen Beziehungen in der medialen Strategie betont. Den langjährigen Kontakten und gewachsenen Beziehungen von einzelnen Personen in politische Institutionen und in Organisationsnetzwerken wird eine herausragende Bedeutung zugemessen (Interview 11, 5, 26). Lobbying und Interessenvermittlung sind ein auf persönlichen Beziehungen beruhendes Geschäft. Sollte eine zentrale Person in dieser Rolle wegfallen, ist dies für Organisationen äußerst schwer zu kompensieren, da solche Beziehungen langfristig aufgebaut werden müssen (Interview 11).

\subsection{Auswertung der materialbasierten Kategorie (4) Legitimationsargumentation}

Das vierte und letzte Kategorienset thematisiert die Überlegungen zur Legitimationsargumentation von NGOs. Das Set gliedert sich in die Kategorien der Legitimation durch basisdemokratische, advokatorische, moralisch-idealistische, outputlegitimierte, thematische, legalistisch-juristische sowie transparenzbezogene Argumente (Abbildung 5.13). Explizit soll an dieser Stelle darauf hingewiesen werden, dass im folgenden Abschnitt die erfragten Legitimationsargumente der Interviewpartner*innen analysiert werden. Auch wenn es in den Antworten häufig anklingt, kann an dieser Stelle keine demokratietheoretische Debatte begonnen werden, die eine qualitative Bewertung der genannten Argumente mit sich bringen würde. Fremd- und Selbstzuschreibungen sowie Erwartungen an die Organisationen werden benannt, aber nicht bewertet. Die etablierten Konzepte 
sind nicht gleichzusetzen mit universellen Kriterien, die einer Organisation die Legitimation verschaffen, im politischen Willensbildungs- und Gesetzgebungsprozess mitzuwirken. Vielmehr wird diese in einem breiten Konsens des Mainstreams der Politikwissenschaft in Form des prozeduralen Gemeinwohls in pluralistischen Gesellschaften als grundsätzlich gesetzt angesehen (ausführlich in Strünck 2014, siehe Kapitel 6).

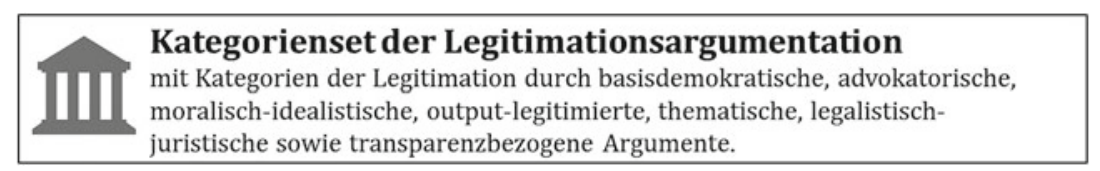

(Grafik: eigene Darstellung)

Abbildung 5.13 Kategorienset der Legitimationsargumentation

Im Folgenden wird die Bandbreite an genannten Legitimationsargumenten aufgezeigt, die sich an den ausgewählten NGOs sowie einem weiten Kreis an zivilgesellschaftlichen Organisationen festmachen lassen. Die Legitimationsargumente sind nicht starr, sondern Spiegel des Zeitgeistes und der Veränderung von strukturell bedingten Sichtweisen (siehe Legitimationsmuster „Transparenz und Selbstwirksamkeit“). Generell zeigt sich, dass die Diskussion um Legitimation von NGOs erschwert wird, weil unterschiedliche Bezugsrahmen vermischt und die (impliziten) Dimensionen des jeweiligen Legitimitätsverständnisses nicht klar voneinander abgegrenzt werden. Auch innerhalb der NGO-Szene wird der Unterschied zwischen Interessenaggregation klassischer Mitgliederstrukturen und anderen Legitimationsarten diskutiert. Denn anders als in der idealtypischen Rückkopplung von Parteien oder Mitgliedschaftsverbänden an ihre formale Mitgliederbasis, betrachten NGOs, laut Interviewaussagen, innerverbandliche Basisdemokratie nicht als essenzielle Legitimationsgrundlage (Interview 8, 21, 30, 26, 29).

Auf Grundlage der Interviews konnten sieben Legitimationsmuster rekonstruiert werden. Diese schließen sich nicht gegenseitig aus, sondern skizzieren Legitimationsargumente von Selbstwahrnehmung und Selbstvergewisserung, bis hin zu strategischen Überlegungen und grundrechtlich verbürgten Rechten. So wird Legitimation jenseits der beiden Eckpunkte von advokatorischer und basisdemokratischer Begründungen über thematische, moralisch-idealistische, legalistischjuristische Legitimationsargumente, Output-Legitimation und Argumente der Transparenz und Wirksamkeitserfahrung konstruiert. 


\section{Basisdemokratische Legitimation}

Grundlage für dieses Legitimationsmuster ist die idealtypische Vorstellung einer basisdemokratisch abgesicherten und mandatierten Interessenvertretung. Demnach setzt sich eine Organisation für die aggregierten Interessen der eigenen Mitglieder ein. Diese idealtypische Vorstellung muss mehrfach relativiert werden. Einen vermeintlichen, arithmetisch gebildeten Mehrheitswillen der Bevölkerung abzubilden, ist nicht das Ziel der Organisationen. Es wird vereinzelt sogar als arrogant eingeschätzt, dies zu behaupten (Interview 22, 17). Einige Organisationen haben basisdemokratische Strukturen aufgebaut und werden vom Engagement und den finanziellen Beiträgen der Mitglieder inhaltlich getragen. Sie konstituieren ihre Stärke und Einfluss auch über die Anzahl ihrer Mitglieder (siehe Abschnitt 5.1; Interview 26, 19). Die Mitglieder sind ein zentraler Identifikationspunkt für die eigene Tätigkeit, ohne die die Organisation auch nicht arbeitsfähig wäre (Interview 5, 12). Diese Organisationen sind häufig als Grassroots-Organisationen aus Bewegungen entstanden (Interview 26) und haben eine große Strahlkraft über die enge Mitgliedschaft hinaus (Interview 9).

Die Legitimation über reine Zahlen wird allerdings auch von diesen Organisationen vielfach kritisch gesehen, da die Intensität der Unterstützung oftmals unklar bleibt. Gemeint sind hier zahlende, aber inaktive Mitglieder sowie das Gleichsetzen von Unterstützung mit Clicks oder Newsletterabonnent*innen (Interview 6). Dennoch wird die Darstellung einer großen Unterstützerschaft als Hebel für politische Einflussnahme gesehen (Interview 10). Vielfach konnte festgestellt werden, dass andere Legitimationsargumente zusätzlich durch die basisdemokratische Mitgliedschaft untermauert wurden (Interview 16).

\section{Advokatorische Legitimation}

Dieses Legitimationsmuster greift die idealtypische Vorstellung auf, das Organisationshandeln über die Tätigkeiten im Namen bzw. im Interesse bestimmter Bevölkerungsgruppen zu rechtfertigen. Ausgangspunkt ist die schlechtere Organisations- und Konfliktfähigkeit von „schwachen Interessen“, die sich selbst nicht oder nicht ausreichend vertreten können (bspw. Menschenrechts-, Umweltund Verbraucherschutzthemen, marginalisierte gesellschaftliche Gruppen, demokratische Strukturen; Interview 10, 11, 30, 21, 8, 6). Für das Selbstverständnis und die Handlungsmotivation der Organisationen ist die Wahrnehmung legitimierend, ein Anliegen zu vertreten, welches andernfalls keine Lobby hätte (Interview 14). Damit werden auch die Mitteleinwerbung und Unterstützungsaufforderung gerechtfertigt: Wer möchte, dass diese Interessen auch weiterhin stellvertretend professionell vertreten werden, muss sich finanziell einbringen (Interview 27). In 
Teilen kann die organisationale Arbeit über den Umweg einer breiten, advokatorischorientierten Beteiligung zusätzlich bottom-up unterstützt werden (Interview $6)$.

\section{Moralisch-idealistische Legitimation}

Die moralisch-idealistische Selbstwahrnehmung, als Organisation die (eigene) Vision einer gerechteren Welt umzusetzen, ist ein gängiges Legitimationsmuster. Es richtet sich an der subjektiven Überzeugung aus, auf der ,richtigen Seite“ zu stehen (Interview 6) und zu ,den Guten“ dazuzugehören (Interview 6, 21). Die Argumentation unterteilt sich in die Vertretung von - legitimen, aber privaten - Partikularinteressen, dem Einsatz für breite Bevölkerungsteile bis hin zum Engagement für zentrale „Gemeinwohlinteressen“ (Interview 5, 2). Der Begriff des Gemeinwohls - unscharf und mit Fremdzuschreibungen überlagert - ist in diesem Kontext Legitimationsgrundlage und Hauptkritikpunkt (siehe Kapitel 6). Analytisch aufgearbeitet lassen sich verschiedene Gemeinwohl-Bezugspunkte als Argumentationsgrundlage identifizieren.

Zunächst ist das prozessorientierte Ideal zu nennen. Es provoziert eine permanente normative Reflexion über die Frage, wie fairer Interessenausgleich in politischen Entscheidungsprozessen gestaltet werden kann, sodass die Grundanliegen von möglichst vielen Menschen geteilt werden (Interview 26). Durch das Ideal der aufklärerisch-aktivierenden Partizipation sehen sich Organisationen selbst in der Pflicht für demokratische Partizipation zu werben, damit möglichst plurale Interessen in den politischen Prozess eingespeist werden können (Interview 26). Mit dem zweiten Ideal ist auch inhaltliche Informationsarbeit verbunden. Dies folgt der Überzeugung, dass Organisationen Forderungen stellen sollen, die gesamtgesellschaftlich akzeptiert sein müssen (Interview 11). Das dritte Ideal unterscheidet zwischen Partikularinteressen und Gemeinwohlinteressen. Es orientiert sich an der stark vereinfachenden, vielfach kritisierten Aufteilung von Lobbyist*innen auf der einen Seite und Gemeinwohlvertreter*innen auf der anderen Seite. Diese Komplexitätsreduktion wird auch unter NGOs teilweise kritisch gesehen (Interview 15, 30, 3).

\section{Output-Legitimation}

Die Output-Legitimation entsteht klassischerweise durch das Erreichen politischer Ziele. Das organisationale Handeln wird öffentlichkeitswirksam aufbereitet, um es möglichst effizient in die Medien zu bringen (Interview 29, 15, 3, 9). Einschränkend ist jedoch, dass die tatsächliche Umsetzungsbilanz bzw. die Grenzen der politischen Wirksamkeit der Organisationen häufig eher schmal ausfallen (siehe Abschnitt 5.1). Dies begrenzt die Legitimation über den eigenen Output im 
Sinne umfangreicher politischer Veränderungen (Interview 30). Allerdings werden auch die Leistungen von Organisationen anerkannt, die als kritische Stimme die demokratischen Strukturen schützen möchten. Diese Zuschreibung kann ebenfalls legitimitätsstiftend sein (Interview 16; siehe Abschnitt 5.1).

\section{Thematische Legitimation}

Für die Legitimationszuschreibung spielt thematische Fachexpertise in einem gesellschaftlich als relevant anerkannten Bereich eine essenzielle Rolle. Organisationen werden Kompetenzen und Relevanz zugesprochen, wenn es ihnen gelingt, eigenes Fachwissen professionell, transparent und kontinuierlich zu generieren (Interview 30). Diese glaubwürdige, anerkannte und inhaltlich fundierte Expertise aus der jahrelangen Beschäftigung mit den Kernthemen - gleichermaßen bei NGOs, Verbänden und anderen Akteuren der Interessenvermittlung - wird als thematische Legitimation verstanden (Interviews 30, 26). Die Zuschreibung der Themenrelevanz ist nicht objektiv, sondern geschieht u. a. über gesellschaftliche Wahrnehmung des Themas (Interview 29, 26), über das investigative Aufdecken eines bisher wenig beachteten Problemfelds (siehe Abschnitt 5.5) und kann auch durch die Organisationen selbst beeinflusst werden.

\section{Legalistisch-juristisch}

Das legalistisch-juristische Legitimationsmuster richtet sich an die im Grundgesetz verbürgten Grundrechte der Meinungs-, Vereinigungs- und Versammlungsfreiheit (Interview 8, 19). Diese idealtypische Argumentation schließt zunächst alle inhaltlichen oder normativ-moralischen Aspekte der Interessenvertretung aus und orientiert sich streng an formalistischen Idealen. Damit stellt sich dieses Muster argumentativ vor die politische Debatte, wer welche Interessen vertreten darf (Interview 8, 19). Eine moralisch-idealistische Note erhält dieses Legitimationsmuster über den Bezug zu den Menschenrechten. Es umschließt die Verteidigung der Würde des Menschen, um die Notwendigkeit zum Handeln herzuleiten (Interview 19).

\section{Transparenz und Selbstwirksamkeit}

Die Transparenzanforderungen an NGOs wachsen im Zeitalter der Informationsgesellschaft nicht nur aufgrund vergangener Spendenskandale immer weiter an. Zum Ausdruck kommt dies auch durch die freiwillige Selbstverpflichtung von rund 1.200 Organisationen (darunter Foodwatch, LobbyControl und Campact, nicht aber Attac und die Deutsche Umweltstiftung) den zehn Vorgaben 
der Initiative Transparente Zivilgesellschaft ${ }^{1}$ zu entsprechen. Die Forderung, über Ursprung und Verwendung von Mitteln, die genauen Ziele der Organisationen, aber auch über Transparenz innerorganisationaler Entscheidungsstrukturen oder Interessenskonflikte zu informieren, wird nicht nur von den Unterstützer*innen, sondern häufig von den Organisationen selbst gestellt (Interview 3, 28).

Diese Transparenzerwartungen sind eng an die Darstellung von organisationalen Erfolgen und Wirkung gekoppelt. Wer als Organisation glaubhaft machen kann, dass der eventuelle Organisationserfolg direkt auf die finanzielle Unterstützung oder das individuelle ehrenamtliche Engagement zurückzuführen ist, kann Legitimation indirekt über die Selbstwirksamkeitserfahrung der jeweiligen Unterstützer*innen erhalten (Interview 3, 12). Zu unterscheiden ist dieser Legitimationsaspekt von der klassischen Output-Legitimation, die sich auf Legitimation durch Organisationserfolg bezieht. Kern des Legitimationsmusters „Transparenz und Selbstwirksamkeit“ ist die (Selbst-)Wirksamkeitserfahrung jeder individuellen Unterstützer*in.

\section{Legitimationsmuster der untersuchten NGOs}

Attac hat als einzige der untersuchten NGOs eine ausgeprägt basisdemokratische Mitgliederstruktur. Die Mitglieder stehen im Zentrum und sind identitäts- und legitimationsstiftend. Auch die thematische und strategische Ausrichtung wird bottom-up entschieden. Darüber hinaus besteht die klare Vorstellung sich für eine bessere, gerechte Welt einzusetzen. Das basisdemokratische Legitimationsargument ist daher eng mit dem moralisch-idealistischen Argument, sowie in Teilen mit dem advokatorischen verbunden.

LobbyControl kann zunächst das expertisebezogene, thematische sowie das advokatorische Legitimationsargument zugeschrieben werden. Ausschlaggebend ist die Wahrnehmung, unterrepräsentierte Interessen zu vertreten, die sich selbst nicht (gut) organisieren können. Verbunden damit ist die thematische Legitimation über die eigene ihre Rolle als Fachexperte in einem gesellschaftlich als relevant angesehenen Problemfeld. Die Organisation selbst führt zudem den Wunsch nach Legitimation über den organisationalen Output an. In kritischer Selbstwahrnehmung ist sich LobbyControl aber bewusst, dass sie bisher nur wenige Kernforderungen erreichen konnten (siehe Abschnitt 5.1).

\footnotetext{
${ }^{1}$ Die Initiative Transparente Zivilgesellschaft ist angegliedert an Transparency International e.V. und wurde 2010 nach dem Unicef Spendenskandal durch ehrenamtliche Mitarbeiter*innen ins Leben gerufen, um solchen Ereignissen vorzubeugen, aber auch um grundsätzlich die Informationslage über zivilgesellschaftliche Gruppen zu verbessern (Initiative Transparente Zivilgesellschaft 2019).
} 
Foodwatch nutzt als Legitimationsbasis explizit die legalistisch-juristische Argumentation. Die Organisation weist ausdrücklich und proaktiv darauf hin, dass den zivilgesellschaftlichen Organisationen die grundrechtlich verbürgten Rechte der Meinungs-, Vereinigungs- und Versammlungsfreiheit zustehen. Verbunden ist diese Argumentation mit der moralisch-idealistischen Vorstellung, sich für Bürgerrechte stark zu machen. Hinzu kommen Aspekte der advokatorischen Argumentation, sich für Themen einzusetzen, die sich selbst nur schwer organisieren können - wie individuelle Verbraucher*innen gegen Unternehmen.

Bei Campact muss die Legitimationsargumentation dreigeteilt werden. In der eigenen Überzeugung liegt eine basisdemokratische Vorstellung vor: Die Organisation stellt die Infrastruktur bereit, in der Menschen die Möglichkeit haben, sich für ein Thema einzusetzen. Eng damit verbunden ist die Selbstwirksamkeitserfahrung der Unterstützer*innen. Der zweite Teil orientiert sich am Erfolgskriterium, nach dem sich die Organisation auf politische Entscheidungen konzentriert, die auf der Kippe stehen. Dies fällt in die Output-Legitimation, in der die erzielten Erfolge für die Unterstützer*innen das Organisationsselbstverständnis prägen. Der dritte Teil richtet sich an das Ziel eines sozialen, ökologischen und demokratischen Fortschritts, der trotz des strukturellen Nachteils zwischen starken und schwachen Interessen erreicht werden soll. Mit dem Wunsch nach gesellschaftlicher Veränderung ohne definiertem Kernthemenset entspricht dies der moralisch-idealistischen Argumentation.

Bei der Deutschen Umweltstiftung lässt sich - auf Grundlage des beschränkten Datenmaterials - lediglich eine Vermutung formulieren. Die Legitimitätsargumentation erfolgt hier moralisch-idealistisch: über die Ziele zu den übergeordneten Themen Umwelt und nachhaltigen Konsum, die für die Gesellschaft erreicht werden sollen sowie über die Vorstellung, gesellschaftliche Veränderungsprozesse über individuelle Verhaltensänderung zu erreichen.

\section{NGOs als Projektionsflächen - Selbst- und Fremdwahrnehmung}

NGOs werden häufig zu Projektionsflächen von Medien, Politik und anderen Interessengruppen. Fremd- und Selbstwahrnehmung von Funktion und Möglichkeiten der NGOs divergieren zum Teil dramatisch. Die Zu- oder Aberkennung von Legitimation ist häufig abhängig von sich (temporär) überschneidenden Interessen von politischen Akteuren, Medien und NGOs sowie von der medialen bzw. politischen Inszenierung als Gegenspieler. Dies hängt vor allem mit der grundsätzlichen strategischen Aufstellung und dem eigenen Selbstverständnis zusammen. 
Aufgrund der Unterschiede zu etablierten Mitgliedschaftsverbänden herrschen unterschiedliche Vorstellungen über die Einbindung von NGOs in eher klassischkorporativ geprägte Formate des politischen Betriebs vor. In Multi-StakeholderKonstellationen zählt nicht mehr nur das das Kriterium einer hinreichenden Mitgliederstärke, um an den Verhandlungstisch gelassen zu werden. Allerdings ist die vorherrschende Expert*innenmeinung, dass es eine gewisse Form von Organisationsgrad braucht, um politische Relevanz zu entfalten. Denn nur mit einem gefestigten Organisationskern können die speziellen Interessen im politischen Willensbildungsprozess nachhaltig aufgenommen werden (Interview 10).

Insgesamt findet auch innerhalb der NGO-Landschaft eine Reflexion der eigenen Rolle statt. Diese bezieht sich auf ihre eigenen Funktionen und Rollen innerhalb der Zivilgesellschaft, die grundsätzliche Rolle und Funktion von Zivilgesellschaft, den eigenen Arbeitsmodus sowie auf vorherrschende Legitimationsargumente der eigenen Organisation, aber auch anderer NGOs. Kritisch wurde teilweise der Arbeitsmodus von reinen Online-Petitionsplattformen gesehen, wenn eine Rückkopplung an Offline- Aktivitäten fehlt.

\section{Zusammenfassung}

Das Kapitel präsentierte die Ergebnisse der qualitativen Analyse anhand der theoriegeleiteten und der materialbasierten Kategorien. Die fünf ausgewählten NGOs wurden zunächst innerhalb des Dreiecks der Handlungsrationalität mit ihren Positionsausrichtungen in der Unterstützungs-, Einfluss- und Reputationslogik verortet. Ergänzende Schwerpunkte in den Handlungsstrategien wurden über die Kategorien der übergeordneten Strategie, der Themenwahl, der Rolle von Expertise und der Legitimationsargumentation gesetzt. Die Ergebnisse werden im Folgenden hinsichtlich ihrer praktischen Implikationen bewertet, bevor das hypothesengenerierende Fazit der Untersuchung gezogen wird. 
Open Access Dieses Kapitel wird unter der Creative Commons Namensnennung 4.0 International Lizenz (http://creativecommons.org/licenses/by/4.0/deed.de) veröffentlicht, welche die Nutzung, Vervielfältigung, Bearbeitung, Verbreitung und Wiedergabe in jeglichem Medium und Format erlaubt, sofern Sie den/die ursprünglichen Autor(en) und die Quelle ordnungsgemäß nennen, einen Link zur Creative Commons Lizenz beifügen und angeben, ob Änderungen vorgenommen wurden.

Die in diesem Kapitel enthaltenen Bilder und sonstiges Drittmaterial unterliegen ebenfalls der genannten Creative Commons Lizenz, sofern sich aus der Abbildungslegende nichts anderes ergibt. Sofern das betreffende Material nicht unter der genannten Creative Commons Lizenz steht und die betreffende Handlung nicht nach gesetzlichen Vorschriften erlaubt ist, ist für die oben aufgeführten Weiterverwendungen des Materials die Einwilligung des jeweiligen Rechteinhabers einzuholen. 